\title{
SH3KBP1 scaffolds endoplasmic reticulum and controls skeletal myofibers architecture and integrity
}

Alexandre Guiraud ${ }^{1 *}$, Emilie Christin ${ }^{1 *}$, Nathalie Couturier ${ }^{1 *}$, Carole Kretz-Remy ${ }^{1}$, Alexandre Janin1, Alireza Ghasemizadeh¹, Anne-Cécile Durieux², David Arnould ${ }^{2}$, Norma Beatriz Romero3 ${ }^{\text {, Mai Thao Bui }}{ }^{3}$, Vladimir L. Buchman ${ }^{4}$, Laura Julien5, Marc Bitoun $^{5}$ and Vincent Gache ${ }^{1}$

$11 *$ Contribute equally

12 1-U1217, UMR 5310, INMG, INSERM, CNRS, Claude Bernard University Lyon 1, France.

13 2- Laboratoire Interuniversitaire de Biologie de la Motricité, Université de Lyon, Université 14 Jean Monnet, Saint Etienne, France.

15 3- Unité de Morphologie Neuromusculaire, Institut de Myologie, Groupe Hospitalier 16 Universitaire La Pitié-Salpêtrière, Paris, France.

17 4- School of Biosciences, Cardiff University, Museum Avenue, Cardiff, CF10 3AX, UK.

18 5- Sorbonne Université, INSERM, , Institute of Myology, Centre of Research in Myology, UMRS 19 974, F-75013, Paris, France.

\section{Summary}

Myonuclei are actively positioned throughout muscular development. Guiraud, Christin, Couturier et al show that SH3KBP1 scaffolds the ER through Calnexin interaction and controls myonuclei motion during early steps of muscle fibers formation. Besides SH3KBP1 participates in cell fusion and T-tubules formation/maintenance in mature skeletal muscle fibers and contributes to slow-down CNM-like phenotypes.

\section{Abstract}


myonuclei are actively positioned throughout muscular development and adopt special localization in mature fibers: regular spacing along muscle fibers periphery, raising the notion of MyoNuclear Domains (MNDs). There is now growing support for a direct connection between myonuclear positioning and normal function of muscles, but how myonuclei affects muscle function remains poorly characterized.

To identify new factors regulating forces applied on myonuclei in muscles fibers, we performed a siRNA screen and identified SH3KBP1 as a new factor controlling myonuclear positioning in early phases of myofibers formation. Depletion of SH3KBP1 induces a reset of MNDs establishment in mature fibers reflected by a dramatic reduction in pairwise distance between myonuclei. We show that SH3KBP1 scaffolds Endoplasmic Reticulum (ER) in myotubes that in turn controls myonuclei velocity and localization and thus myonuclear domains settings. Additionally, we show that in later phases of muscle maturation, SH3KBP1 contributes to the formation and maintenance of Sarcoplasmic Reticulum (SR) and Transverse-tubules (T-tubules). We also demonstrate that in muscle fibers, GTPase dynamin-2 (DNM2) binds to SH3 domains of SH3KBP1. Interestingly, we observed that Sh3kbp1 mRNA is up regulated in a mouse model harboring the most frequent mutation for Autosomal Dominant CentroNuclear Myopathy (AD-CNM): Dnm2+/R465W. SH3KBP1 thus appears as a compensation mechanism in this CNM model since its depletion contributes to an increase of CNM-like phenotypes (reduction of muscle fibers Cross-section Areas (CSA) and increase in slow fibers content). Altogether our results identify SH3KBP1 as a new regulator of myonuclear domains establishment in the early phase of muscle fibers formation through ER scaffolding and later in myofibers integrity through T-tubules scaffolding/maintenance.

\section{Introduction}

The building block of skeletal muscle is the multinucleated muscle fiber, formed by the fusion of hundreds of specialized cells, myoblasts, and in which positioning of nuclei ("myonuclei") is finely regulated. In the normal course of muscle development or regeneration, myonuclei actively localize themselves and adopt specific position in mature myofibers in which they are regularly spaced at the periphery of myofibers (Bruusgaard et al, 2003). This precise myonuclei spatial organization gives rise to the notion of MyoNuclear Domains (MNDs) in which each myonucleus controls genes expression in its surrounding cytoplasm and guaranties muscle function 61 (Gundersen, 2016). MNDs settings mainly depend on muscle fibers ability to maintain a defined distance between myonuclei in a cytoplasmic-adapted context related to myofibers type, size and age (Bruusgaard et al, 2006; Qaisar \& Larsson, 2014; Liu et al, 2009). During the first steps of muscle fibers formation, myonuclei have been shown to move and position using mainly microtubule network reorganization (Tassin et al, 1985; Gimpel et al, 2017) and an interplay between microtubule associated proteins (MAPs) such as MAP7 (Metzger et al, 2012) and molecular motors, including dynein and kinesins (Gache et al, 2017). This dynamic of myonuclei contributes to precocious alignment of myonuclei in immature fibers. Although comprehension of mechanisms involved in myonuclear positioning during myofibers formation has recently progressed, how mispositioning of myonuclei affects muscle function still remains an open question. 
Bitoun, 2011; Jungbluth et al, 2007). The majority of defective proteins implicated in CNMs are involved in various aspects of membrane trafficking and remodeling and are relevant to essential cellular processes including endocytosis, intracellular vesicle trafficking and autophagy (Romero \& Laporte, 2013). To date, the main proteins implicated in CNMs are phosphatidylinositol phosphatase Myotubularin coded by MTM1 gene (Laporte et al, 1996); GTPase dynamin-2 involved in endocytosis and cell motility, and coded by DNM2 gene (Bitoun et al, 2005); amphiphysin-2 nucleocytoplasmic adaptor protein, coded by BIN1 gene (Nicot et al, 2007) and the principal sarcoplasmic reticulum calcium release channel, ryanodine receptor 1, coded by $R Y R 1$ gene (Wilmshurst et al, 2010; Bevilacqua et al, 2011).

Myonuclei spatial organization can contribute to muscle fibers functionality as growing evidences support a direct connection between myonuclear positioning and normal function of muscles (Metzger et al, 2012; Falcone et al, 2014; Janin \& Gache, 2018; Robson et al, 2016). Additionally, T-tubule organization and efficiency are highly impacted in CNM (Chin et al, 2015; Al-Qusairi et al, 2009). Finally, besides abnormal nuclear positioning and T-tubules abnormalities, altered autophagy is frequently described in CNM (Jungbluth \& Gautel, 2014).

SH3KBP1, also known as Ruk/CIN85 (Cbl-interacting protein of $85 \mathrm{kDa}$ ) is a ubiquitously expressed adaptor protein, involved in multiple cellular processes including signal transduction, vesicle-mediated transport and cytoskeleton remodeling (Havrylov et al, 2010; Buchman et al, 2002). SH3KBP1 protein is composed of three SH3 domains at the N-terminus followed by a proline-rich (PR) domain, a serine-rich (SR) domain, and a Cterminus coiled-coil (CC) domain. Functions of SH3KBP1 as an adaptor protein are mainly linked to endocytosis trafficking and degradative pathway through the recruitment of Cbl (E3 ubiquitin ligase), endophilin and dynamin (Schroeder et al, 2010; Sun et al, 2015; Zhang et al, 2009). SH3KBP1 protein is also associated with several compartments involved in membrane trafficking such as the Golgi complex and is mainly concentrated in COPI-positive subdomains (Havrylov et al, 2008). The role of SH3KBP1 during the formation of muscle fibers has never been investigated.

In the present study, we show that the adaptor protein SH3KBP1, through its N-terminus part, is able to scaffold endoplasmic reticulum (ER) probably via an interaction with Calnexin. SH3KBP1 also governs myonuclei motion and spatial organization as well as myoblast fusion. Additionally, we observed that SH3KBP1 interacts with Dynamin 2 (DNM2) and organizes T-tubule formation in skeletal muscle. Moreover, its down-regulation contributes to an increase of CNM-like phenotypes in a mouse model expressing the most frequent mutation causing autosomal dominant centronuclear myopathy (AD-CNM).

\section{Results}

103 SH3KBP1 is required for precocious myonuclear positioning steps and controls fusion parameters during muscle fiber formation.

To identify new factors that contribute to myonuclear spreading and alignment in muscle fibers, we performed a large siRNA screen on candidates expected to affect myonuclei repartition in nascent myotubes. Briefly, primary myoblasts were isolated from young pups and induced to differentiate in vitro for 3 days before analysis (Falcone et al, 2014) (Fig.1A). This cell culture technique leads to an accumulation of heterogeneous myotubes regarding 
myonuclei number and myotubes length (Blondelle et al, 2015). To precisely characterize the early steps of 111 myonuclei positioning in myotubes, we developed an image-J® ${ }^{\circledR}$ plugin that automatically extracts different myotubes parameters (such as myotubes lengths/areas and myonucleus localization). Data were then classified and analyzed according to myonuclei content using a homemade program in R-Studio ${ }^{\circledR}$ (Fig.1D-F). This analysis allowed us to track myonuclei accretion during elongation of myotubes in a window from 3 to 11 myonuclei per myotubes. Isolated murine myoblasts were then treated with short interfering RNA (siRNA) targeting either a scrambled sequence or candidate genes. Among candidates from the siRNA screen, efficient sh3kbp1 depletion appears to strongly modify myonuclear positioning (Fig1. B-C, Fig.S1A). In control condition, we observed a nearly linear relation between addition of myonuclei into myotubes and expansion of myotubes length, with an average of nearly $10 \%$ increase of length after each myonucleus accretion (Fig. 1D). In sh3kbp1 depleted myotubes, length repartition is homogenously extended to an increase of $46.8 \% \pm 3.3$ in myotubes containing up to 11 myonuclei (Fig. 1D). Quantification of the mean distance between all myonuclei inside myotubes indicate a burst of spacing $(+103.5 \% \pm 11.6$, data not shown) concomitantly with an escape from the center of myotubes reflected by (i) the mean distance between myotube centroid and each myonuclei (DMcM) $(+111.5 \% \pm 12.5)$ (Fig. 1E) and (ii) the Myonuclei Spreading Graphic (MSG) representation (Fig. 1F). MSG shows statistical probability to find one myonucleus along the all length of myotubes and allow the extraction of "statistical clustering zones" (colors code in Fig. 1F) that we estimate as four zones in the case of scramble myotubes versus eight zones in sh3kbp1 depleted myotubes (Fig. 1F). Thus, sh3kbp1 depleted myotubes failed to homogeneously spread myonuclei along myotubes length that tends to accumulate at myotubes tips (Fig. 1C, arrows). Overall, these data suggest that for a defined myonuclei content in myotubes, SH3KBP1 acts as "anti-elongation" factor and contributes to myonuclei spreading in myotubes.

131 Myoblasts/myotubes elongation and alignment is a key process that controls myoblast/myotubes fusion (Louis et al, 2008). As sh3kbp1 depletion led to myotubes length increase, we wondered if fusion aspects were modified during differentiation. Indeed, myotubes elongation can contribute to increase myotubes/myoblast contacts that thus will modulate membrane fusion and ultimately modify myonuclei accretion speed (Kim et al, 2015). We evaluated fusion capacity of primary myoblasts isolated from young pups after in vitro differentiation induction (Fig.S1B-G). After 3 days of differentiation, isolated myoblasts treated with siRNA targeting sh3kbp1 mRNA are similarly stained for myosin heavy chain antibodies compare to control cells treated with siRNA scramble sequences, indicating normal myoblast commitment into muscle differentiation process (Fig. S1B-D). Fusion index was increased in Sh3kbp1 depleted myotubes, assessed by the total number of myonuclei in myotubes (Fig. S2E) and the average number of myonuclei per myotube (Fig. S2F). Accordingly, in sh3kbp1 depleted conditions, distribution of myotubes with respect to myonuclei content revealed much more myotubes with $\geq 10$ nuclei when compared to controls (Fig. S2G). Altogether, this data suggests that sh3kbp1 acts also as an "anti-fusion" factor as expected for an anti-elongation factor.

SH3KBP1 governs myonuclei velocity that contributes to myonuclei positioning in mature myofibers. 
In 5-day differentiated myofibers, myonuclei clustering and velocity was addressed (Fig. 2B-F). First, we observed twice more myonuclei clustered in myotubes treated with shRNA targeting sh3kbp1 gene compared to control, confirming a role of sh3kbp1 on myonuclei spreading (Fig. 2B \& E, white asterisk). As myonuclei within myofibers have different behaviors (Gache et al, 2017), we next investigated the impact of sh3kbp1 depletion on myonuclei movements in 5-day differentiated myofibers. Myoblasts were transfected with RFP-laminchromobody ${ }^{\circledR}$ to visualize myonuclei concomitantly with shRNA targeting scramble or Sh3kbp1, both GFPtagged. Myotubes containing both constructions (GFP and RFP-lamin-chromobody ${ }^{\circledR}$ ) were selected for fewer analyses (Fig.2C-F). After 5 days of differentiation, myonuclei were tracked every 20 minutes for a time period of 16 hours (Fig. 2E, Supplementary video 1-2). Myonuclei displacements parameters were analyzed using SkyPad method (Cadot et al, 2014). We found that in control condition, myonuclei are in motion during nearly $35 \%$ of the time (a movement is defined as a displacement more than $30 \mu \mathrm{m}$ ) at a median speed of $0.232 \pm 0,014$ $\mu \mathrm{m} / \mathrm{min}$. Depletion of sh3kbp1 increased from more than $20 \%$ the percentage of time myonuclei are in motion of and by more than $30 \%$ median speed that reach $0.313 \pm 0,014 \mu \mathrm{m} / \mathrm{min}$ outside myonuclei clusters (Fig.2C-D). Indeed, because of high myonuclei concentration inside myonuclei clusters, we could not technically access to myonuclei motion and speed (Fig. 2E, Supplementary video 2). To further investigate the implication of sh3kbp1 in late steps of differentiation, primary mouse myotubes were maintained in differentiation media for 10 days as previously described (Pimentel et al., 2017) (Fig. 2G). In these maturation conditions, myonuclei are compressed between myofiber plasma membrane and contractile apparatus and adopt a flatten architecture all along myofibers length (Roman et al., 2017). This long-term differentiation approach allow to confirm that sh3kbp1 controls myonuclear positioning during maturation of myofibers as sh3kbp1 depletion using either siRNA or shRNA caused a significant reduction in the mean distance between adjacent myonuclei (Fig. 2G-H). Additionally, Sh3kbp1 depletion significantly reduces by more than $30 \%$ myofibers width (Fig. 2I). These results suggest that SH3KBP1 contributes to nuclear spreading and to the reduction of myonuclei movements and motion during muscle fibers maturation.

Next, we focused on the expression of both SH3KBP1 protein and Sh3kbp1 mRNA in the time course of in vitro myotubes formation using C2C12 cells. These analyses showed a slight enhancement of SH3KBP1 production during early stages of myotubes formation (Fig. 3A). These results were confirmed using RT-qPCR techniques where a two-fold increase was observed in mRNA expression at the onset of differentiation step (Fig3B-C). To confirm Sh3kbp1 functions during muscle cell differentiation, we stably knocked-down Sh3kbp1 mRNA expression in C2C12 cells using a small hairpin interfering RNA (shRNA) (Fig. 3D). As observed in primary cells, after 3 days of differentiation, myoblasts entered the "muscle differentiation program", illustrated by the detection of myosin heavy chain-positive (MHC+) cells (Fig. 3E, day3). Although intensity of staining is reduced, we do not observe any alteration of myotubes size repartition in Sh3kbp1 knocked-down compared to control conditions (Fig. S2A). After 6 days of differentiation, we clearly see a breaking event in fusion capacity correlated with Sh3kbp1 knockdown (Fig. 3E). In control conditions, myonuclei spread along thin myotubes length while Sh3kbp1 knockdown gives rise to huge myotubes with clustered myonuclei areas (Fig. 3E, day6). Distribution of myotubes with respect to their myonuclei content reveals also a significant increase in the number of myotubes with high myonuclei content compared to controls (Fig. S2B). In control condition, we observed a limited accumulation of clustered myonuclei along myotubes length, with a majority of clusters containing 4 to 6 
myonuclei (Fig. 3F). On the opposite, in Sh3kbp1 knockdown, we observe a clear increase in the proportion of clusters containing more than 15 myonuclei (Fig. 3F). To address the specificity of myonuclei clustering phenotype in Sh3kbp1 depleted conditions, we re-expressed full-length SH3KBP1 proteins in Sh3kbp1 depleted murine myotubes and show that we rescue even better than in control the number of clustered myonuclei by myotubes (Fig. S2C-D). Together, these results show that Sh3kbp1 is essential during myotube formation, both in primary myoblasts and in $\mathrm{C} 2 \mathrm{C} 12$ cell cultures, to control myoblasts fusion and myonuclear positioning during skeletal muscle formation.

\section{SH3KBP1 is an endoplasmic reticulum scaffolding protein that interacts with ER72 and Calnexin.}

Several studies show that SH3KBP1 is distributed between different membrane trafficking compartments such as the Golgi Complex (Havrylov et al, 2008). The three SH3 domains localized in the N-terminal part of SH3KBP1 are responsible for its high capacity to interact with diverse regulatory partners (Havrylov et al, 2009) while the C-terminus part, containing a coiled-coil domain allows its targeting to endosomal membranes (Zhang et al, 2009). As no role of SH3KBP1 was previously described in skeletal muscle, we analyzed its localization during the time course of muscle formation using both $\mathrm{C} 2 \mathrm{C} 12$ myoblast cell line and murine primary myoblasts (Fig. 4 A-B). In growing conditions, SH3KBP1 is localized in the cytoplasm with an apparent higher concentration at the vicinity of nuclei both in primary and in C2C12 myoblasts and some dots inside nucleus are also visible (Fig. 4AB). During early myotubes formation, SH3KBP1 seems to spread along myotubes length, exhibit a weak perinuclear accumulation and myonuclei still exhibits dots inside myonuclei (Fig. 4A-B, Day3 and Day2). In 5 days differentiation C2C12 myotubes, perinuclear accumulation of SH3KBP1 is stronger and we still observe some accumulation inside some myonucleus as dots or line (Fig. 4A, Day5). In primary myoblasts induced to differentiate into "mature-like" fibers organized with myonuclei at periphery, SH3KBP1 was still detected at myonuclei vicinity but more accumulated at the interface between myonuclei "bottom" and muscle fiber interior and it also exhibited longitudinal/transversal staining (Fig. 4B, Day10). We thus wondered what kind of compartments could be controlled by SH3KBP1. To answer this question, we used stable C2C12 cell-line depleted for Sh3kbp1 to investigate altered compartment phenotypes (Fig. 4C-D). We observed that, even if myonuclei are clustered in myotubes depleted for Sh3kbp1, Golgi marker RCAS1 was still mainly localized around myonuclei, as previously described for Golgi elements (Fig. 4C)(Ralston et al, 1999). On the contrary, the ER marker ERP72 that showed perinuclear localization in control myotubes was completely dispersed in Sh3kbp1-depleted myotubes (Fig. 4D). This result indicates a role for Sh3kbp1 in the upkeep of ERP72-containing ER specifically at the vicinity of myonuclei, independently of Golgi complex architecture.

ERP72 (also called PDIA4) is a disulfide isomerase that acts as a folding chaperone for newly synthesized secretory proteins in the ER compartment (Satoh et al, 2005). Endogenous ERP72 co-immunoprecipitated with exogenous full-length GFP-tagged SH3KBP1 (Fig. 4E-G). Using a panel of SH3KBP1 deletion mutants, we determined that ERP72 interacts with the C-terminal part of SH3KBP1, independently of its C-terminus coiledcoil domain (Fig. 4E-G). ERP72 is described as an intraluminal ER protein that interacts with calnexin ERchaperone (Penga et al, 2014). Interestingly, the C-terminus part of Calnexin is cytosolic and thus could be the cytoplasmic linker between SH3KBP1 and ERP72-containing Endoplasmic Reticulum (Wada et al, 1991). We 
ERP72, co-immunoprecipitated with exogenous full-length GFP-tagged SH3KBP1 constructs. In addition, we also identified the C-terminal part of SH3KBP1, independently of the C-terminus coiled-coil domain as the Calnexininteracting domain (Fig. 4E-G). Therefore, Proline-and Serine-Rich domains of SH3KBP1 mediate the interaction with ERP72-positive ER through Calnexin binding.

SH3KBP1 progressively accumulates at the Z-line and interacts with DNM2.

We next investigated SH3KBP1 localization in vivo, in mature myofibers from adult skeletal muscle. SH3KBP1 accumulated specifically at the vicinity of myonuclei, forming a "cage" around myonuclei in Tibialis Anterior (TA) muscle (Fig. 5A-B, asterisks). This staining was confirmed in human muscle biopsies where we observed that myonuclei are positive for SH3KBP1 (Fig. S3G). In TA longitudinal section, SH3KBP1 exhibited a striated pattern at the I-band/Z-line zone, in between the staining of the voltage-dependent calcium channel, DHPR $\alpha$, which labels T-tubules and forms a doublet band indicating that SH3KBP1 does not colocalize with DHPR $\alpha$ but follow, in a close proximity, T-tubules structures (Fig. 5C-E). To determine domains of SH3KBP1 responsible for this particular localization, we next tested in vitro expression of full-length SH3KBP1 or associated fragments into primary myoblasts induced to differentiate into "mature-like" fibers with peripheral myonuclei and proper sarcomere organization, reflected by striated actin staining (Fig. 5F-G). In this myofibers, full-length SH3KBP1 was present as small aggregation patches close to myonuclei, combined with striated pattern and accumulated at the Z-line with no overlap with actin staining (Fig. 5F-G, SH3KBP1-FL). N-terminus fragment of SH3KBP1 containing SH3 domains was only present as striated patterns with no particular accumulation at myonuclei vicinity, while the C-terminus SH3KBP1 fragments strongly accumulated at the periphery of muscle fibers, at the vicinity of myonuclei and at the Z-line with striated patterns (Fig. 5.F). Numerous proteins accumulate at the Iband/Z-line zone and participate in myofibers structuration (Burgoyne et al, 2015). Among them, Dynamin 2 (DNM2), a large GTPase implicated in cytoskeleton regulation and endocytosis, was previously described in HeLa cells as a SH3KBP1 interacting protein, through its proline-rich domain (Schroeder et al, 2010). To this end, we expressed, in C2C12 cells, full-length GFP-tagged-DNM2 with fragments of FLAG-tagged SH3KBP1 and immunoprecipitated SH3KBP1 constructs. This experiment confirmed that DNM2 interacts with SH3KBP1 through its N-terminal part (Fig. 5H-I). Therefore, in mature myotubes, SH3KBP1 accumulate at the Z-line where it forms a protein complex with DNM2.

SH3KBP1 is required in mature fibers for the maintenance of T-tubules.

We next assessed the impact of Sh3kbp1 depletion on internal cell architecture. To this end, we used our in vitro model assay on mature fibers using primary myoblasts (Fig. 6A). Myofibrillogenesis in Sh3kbp1 depletion condition was normal after 10 days of differentiation, reflected by F-actin staining (Fig. 6B). In control condition, we also observed that DHPR $\alpha$ staining which labels T-tubule, forms transversal doublet bands alternatively with actin staining (Fig. 6A). Quantification of the staining indicated that in control conditions, using either siRNA or shRNA scramble, nearly $30 \%$ of formed myofibers exhibit a transversal DHPR $\alpha$ staining, reflecting formation of mature T-tubules (Fig. 6A \& C). On the contrary, in Sh3kbp1-depleted myofibers, DHPR $\alpha$ staining is much more punctuated, indicating the absence of mature T-tubules aligned with sarcomere structures (Fig. 6B) and 
myofibers with transversal staining of DHPR $\alpha$ to nearly 5\% of myofibers, correlating with an increase in random DHPR $\alpha$ staining as dots along myofibers (Fig. 6C, Fig. S2E). To confirm these results we depleted Sh3kbp1 from mature myofibers using shRNA electroporation technique directly in TA skeletal muscle of two-month-old mice (Fig. 6D). First, after 15 days of Sh3kbp1 inhibition, an atrophic muscle fiber effect was observed in electroporated TA muscles fibers, reflected by a $35 \%$ decrease of the mean muscle fibers cross section area in Sh3kbp1 fibers compared to controls (Fig. 6F), an effect that we previously observed in vitro (Fig. 2I). Moreover, T-tubule architecture appears perturbed in Sh3kbp1 fibers, illustrated by an unequal DHPR $\alpha$ staining along fibers (Fig. 6D). Together, these results show that Sh3kbp1 contribute to T-tubule formation and maintenance in skeletal muscle fibers.

Sh3kbp1 is up regulated in a murine model of AD-CNM and inhibits CNM phenotypes.

Our data show that Sh3kbp1 controls myonuclei dynamics and reticulum spatial organization and interacts with DNM2 which is mutated in dominant centronuclear myopathy characterized by myonuclear centralization (Romero \& Bitoun, 2011). Heterozygous AD-CNM-Knock-In-Dnm2 ${ }^{\mathrm{R} 465 \mathrm{~W}}$ mouse model (KI-Dnm2 ${ }^{\mathrm{R} 465 \mathrm{~W} /+}$ ) progressively develop muscle atrophy, impairment of contractile properties, histopathological abnormalities including slight disorganization of T-tubules and reticulum, and elevated cytosolic calcium concentration (Durieux et al, 2010). We first analyzed Sh3kbp1 mRNA expression in TA skeletal muscle during mice development and aging (Fig. 7A). To our surprise, Sh3kbp1 mRNA was progressively reduced from more than 30 \% in 8 months old mice compare to 2 months old mice (Fig. 7A), suggesting a progressive loss of SH3KBP1 pool during aging. More interestingly, we also showed that in this $\mathrm{KI}-D n m 2^{\mathrm{R} 465 \mathrm{~W} /+}$ model, Sh3kbp1 mRNA is significantly up-regulated during the first 4 months of mice development (Fig. 7A), suggesting a compensation mechanism, that can explain the limited ratio of atrophic fibers and absence of centralized myonuclei in this CNM model (Durieux et al, 2010). To address the role of SH3KBP1 in long-term muscle homeostasis in both wild type and KI-Dnm2 $2^{\mathrm{R} 465 \mathrm{~W}}$ mice, we investigated in vivo depletion of Sh3kbp1 protein. Down-regulation of Sh3kbp1 was achieved using intramuscular TA muscle injections of an AAV cognate vector expressing shRNA targeting Sh3kbp1 mRNA (AAV-shSh3kbp1) either in wild type or KI-Dnm2 $2^{\mathrm{R} 465 \mathrm{~W}}$ mice at 5 weeks of age, an age where muscle mass is nearly fully developed. This allowed addressing the role of Sh3kbp1 specifically in adult skeletal muscle during the first three months of mice development and specifically in a period where we observed Sh3kbp1 mRNA up-regulation in KI-Dnm2 2465W model (Fig. 7A). In wild type mice, Sh3kbp1 mRNA level was


levels showed a 5.4 fold decrease compared to PBS-injected TA-muscles (Fig. S3A, WT, KI-Dnm2 ${ }^{\mathrm{R} 465 \mathrm{~W}}$ ). No significant change of body weight was observed between AAV-shSh3kbp1-injected and PBS-injected conditions in both genotypes (Fig. S3B). However, a significant decrease in absolute force (g) developed by TA muscles specifically in KI-Dnm2 $2^{\mathrm{R} 465 \mathrm{~W}}$ mice depleted for Sh3kbp1 was observed (Fig. 7D) suggesting a specific impact of Sh3kbp1 down-regulation in the KI-Dnm2 $2^{\mathrm{R} 465 \mathrm{~W}}$ mice model. Interestingly, we observed a significant decrease of TA muscle mass of about $20 \%$, when Sh3kbp1 is depleted in both WT and KI-Dnm2 $2^{\mathrm{R} 465 \mathrm{~W}}$ mice (Fig. 7E). Crosssectional areas of TA-muscle fibers were determined using transverse sections stained with laminin antibodies to define muscle fibers border (Fig. 7B-C). Global decrease in median myofibers area was observed in AAVshSh3kbp1 injected TA-muscles compared to control muscles in WT (-30\%) and KI-Dnm2 ${ }^{\mathrm{R} 465 \mathrm{~W}}(-20 \%)$ mice (Fig. 
7F). Analysis of muscle fibers area repartition showed a significant increase in atrophic fibers in AAV-shSh3kbp1 injected muscles (Fig. S3C). Additionally, we observed a significant reduction of about $25 \%$ in the total number of fibers specifically in KI-Dnm2 $2^{\mathrm{R} 465 \mathrm{~W}}$ model depleted for Sh3kbp1 (Fig. S3D). DNM2-CNM patients exhibit a predominance of type 1 muscle fibers (Romero \& Bitoun, 2011). Thus, we investigated the ratio of slow fiber types in AAV-shSh3kbp1 injected TA-muscles, reflected by the expression of myosin heavy chain type 1 (Fig. 7CD \& G). In wild type conditions, slow fiber type accounted for $5 \%$ of total muscle fibers. We observed that in AAV-shSh3kbp1 injected muscles, this ratio was slightly increased to reach $6.4 \%$ of total muscle fibers, which is the same as what was observed in KI-Dnm2 ${ }^{\mathrm{R} 465 \mathrm{~W}}$ mice (Fig. 7G). However, when Sh3kbp1 is depleted in KIDnm2 $2^{\mathrm{R} 465 \mathrm{~W}}$ muscle, this ratio increases drastically to reach $12 \%$ of total fibers (Fig. $7 \mathrm{G}$ ). In the Knock-in mouse model, DNM2 mutation leads to autophagy impairment (Rabai et al, 2019; Durieux et al, 2010). During autophagosome formation, LC3-I (Microtubule-associated protein 1A/1B-light chain 3) is converted to LC3-II by conjugation to phosphatidylethanolamine lipid. To assess the impact of Sh3kbp1 depletion on autophagy, LC3-II positive fibers was determined in Sh3kbp1 depleted fibers in both WT and KI-Dnm2 ${ }^{\mathrm{R} 465 \mathrm{~W}}$ mice. We observed an increase in number of LC3-II positive fibers in both genotypes but more pronounced in KI-Dnm2 $2^{\mathrm{R} 465 \mathrm{~W}}$ mice (Fig. 7H). Our in vitro data show that Sh3kbp1 depletion increase fusion and alter myonuclei spreading (Fig. 1-3). We thus investigate the impact on the number of myonuclei and the ratio of internalized myonuclei by fibers in Sh3kbp1 depleted myofibers but did not find any evidence for alteration compare to control conditions (Fig. S3EF). Altogether, our data suggest that Sh3kbp1 mRNA increase observed in AD-CNM model could be an attempt of compensatory mechanism as normalization of Sh3kbp1 expression intensified muscle phenotype.

\section{Discussion}

Myonuclei movement's during muscle cell formation have been related to the activity of numerous microtubule binding proteins such as Motors and Maps (Gache et al, 2017). In immature myotubes, microtubules are organized into antiparallel arrays between adjacent myonuclei and contribute to the polarization/elongation of myotubes, alignment of myonuclei and ultimately organization of myosin filaments during sarcomere formation (Pizon et al, 2005; Metzger et al, 2012; Wang et al, 2013). This new orientation/architecture of the microtubule network directly influences myoblasts/myotubes fusiform shape, as microtubule network forces are applied at the extremity of polarized myotubes and also in-between myonuclei (Metzger et al, 2012); it also contributes to myonuclei spreading along myotubes length (Tassin et al, 1985). This precise myonuclei organization in muscle fibers gives rise to the formation of so-called "MyoNuclear Domains" (MNDs) wherein each myonucleus is responsible for gene expression in its surrounding cytoplasm and guaranties functional integrity of muscles (Qaisar, 2012; Liu et al, 2009). However, regulation of processes involved in myonuclei positioning in developing muscle fibers, not directly linked to the microtubule network, are still unknown.

The present study demonstrates that a protein related to the endoplasmic reticulum (ER) network controls both myotubes elongation and myonuclei localization/spreading in developing myofibers, two processes that are closely linked as they both depends on microtubule network organization. Early steps of myonuclei positioning 
2012; Cadot et al, 2012; Wang et al, 2013; Wilson \& Holzbaur, 2014a). This process also depends on myonuclei membrane associated components such as Nesprin-1 or AKAP9 (Gimpel et al, 2017; Wilson \& Holzbaur, 2014b; Doñate Puertas et al, 2018). Here, we show that SH3KBP1 depletion leads to longer and thinner myotubes without significant alteration of the microtubules network compared to control (Fig. 1-3 \& data not shown). We find that the C-terminus part of SH3KBP1 is associated with ERP72-positive ER probably through its binding to Calnexin (Fig. 4E-G). This association scaffolds ERP72-associated ER specifically at myonuclei vicinity, without affecting Golgi apparatus integrity (Fig. 4A-D). Recently, ER has been implicated in governing both microtubule alignment and cytoplasmic streaming (Kimura et al, 2017). Kimura et al propose that local cytoplasmic flow generated along microtubules is transmitted to neighboring regions through the ER and in turn, aligns microtubules and self-organizes the collective cytoplasmic flow. SH3KBP1, by shaping and maintaining ER clustering during myofibers development could contribute to prevent microtubules network anchoring at the nucleus membrane and thus local microtubule network organization. In this view, SH3KBP1, during myoblasts fusion process could disorganize parallel microtubules organization and thus decrease forces applied on myotubes tips extremity. This phenomenon could lead to the control of myotubes length, but also of both myonuclei motility and velocity (Fig. 2).

SH3KBP1 staining is diffuse in dividing cells, while it progressively accumulates at the vicinity of myonuclei during differentiation process (Fig. 4). A few proteins have been shown to form a flexible perinuclear shield that can protect myonuclei from extrinsic forces (Wang et al, 2015; Ghasemizadeh et al, 2019). To note, recent data show that disruption of one component of this perinuclear shield, MACF1 protein, increases myonuclei velocity (Ghasemizadeh et al, 2019). Consequently, SH3KBP1 probably belongs to the group of proteins that contribute to the stability of this perinuclear shield through the maintenance of ER at the vicinity of myonuclei. In accordance with this preferential localization at the proximity of myonuclei, SH3KBP1 staining in transversal Tibialis Anterior muscle section is very intense at myonucleus site whereas nuclei outside the fiber remains negative (Fig. 5A-B). In human muscle biopsies, this localization around myonuclei is maintained even in centralized nuclei from CNM patient (Fig. S3G). The three SH3 domains of SH3KBP1 have been shown to cluster multiple proteins and protein complexes that can also contributes to the stability of those interactions. Havrylov et al identified using mass spectrometry, few microtubule-binding-proteins such as MAP7 and MAP4 that can potentially interact with SH3 domains of SH3KBP1 and have already been shown to control myonuclei positioning in myotubes (Metzger et al, 2012; Mogessie et al, 2015; Havrylov et al, 2009). However, we failed to confirm the interaction of SH3KBP1 with either MAP7 or MAP4 in muscle cells (data not shown). Alternatively, SH3KBP1 also interacts with dynamin-2 (DNM2) and has been shown to participate in dynamics instability of microtubules (Tanabe \& Takei, 2009) and microtubule nucleation (Thompson et al, 2004). The failure of recruiting DNM2 at the right place during myotubes formation after SH3KBP1 depletion could also contribute to the aberrant myonuclei spreading (Fig. 1). Interestingly, in CNM-KI-Dnm2 $2^{\mathrm{R} 465 \mathrm{~W}}$ mouse model, Fongy et al show that myonuclei move and spread properly in heterozygous myotubes but hypothesize a defect in nuclear anchoring at the periphery (Fongy et al, 2019). Several studies pointed the importance of cytoskeleton, including MAPs, microtubules and intermediate filaments, in the nuclear anchorage in mature muscle (Ghasemizadeh et al, 2019; Roman et al, 2017). SH3KBP1 dependent ER-scaffolding could participate in myonuclei anchoring at the 
myonuclei. This hypothesis is supported by our data showing an increase in the percentage of the time in motion of myonuclei, in the absence of SH3KBP1 (Fig. 2D), correlated with the strong staining of myonuclei in mouse and human models (Fig. 5A \& S3G)

In mature myofibers, SH3KBP1 depletion leads to more aggregated myonuclei phenotype, suggesting that a failure in the early phases of myonuclear positioning is difficult to compensate (Fig.2G). Interestingly, sh3kbp1 depletion in long-term primary myofibers culture induces a reduction in myofibers width (Fig. 2I). These results are confirmed in vivo as sh3kbp1 down regulation in Tibialis Anterior muscle reduce from more than $30 \%$ the average cross section areas of myofibers (Fig. 6E-F \& 7F). Moreover, SH3KBP1-depleted mature myofibers show disorganized perinuclear endoplasmic reticulum (ER) (Fig 4). Of interest, ER is one initiation site for autophagic process and ER selective autophagy (called ER-phagy or reticulophagy) has been described to control ER shape and dynamics through ER-phagy receptors that address ER portions to the autophagosomes (Grumati et al, 2018). SH3KBP1 depletion increases the number of LC3II positives myofibers in Tibialis Anterior muscle (Fig. $7 \mathrm{H}$ ), suggesting a possible increase of autophagosomes number. Additionally, SH3KBP1 interacts with dynamin2 (Fig. 5-G), which is also involved in the autophagic lysosome reformation (Schulze et al, 2013). This autophagydependent modulation of muscle homeostasis could first explain the decrease of Cross Section Area and myofibers width that we observed and ultimately the reduction of muscle myofibers (Fig. 2I, 7E-F \& S3C). Interestingly, autophagy genes have been involved in muscle myonuclei positioning during Drosophila metamorphosis (Fujita et al, 2017). Whether this process is involved in SH3KBP1-dependent myonuclei positioning will be the subject of further investigations.

SH3KBP1 depletion shows that T-tubule formation is dramatically impaired both in vitro and in vivo (Fig. 6). Myofibrils provide the contractile force under the control of the 'excitation-contraction coupling' system that includes two membranous organelles: the sarcoplasmic reticulum (SR) and Transverse (T)-tubules (Al-Qusairi \& Laporte, 2011). These two-membrane systems are structurally associated to form the triads of skeletal muscle cells. SR is a complex network of specialized smooth endoplasmic reticulum, essential to transmit the electrical impulse as well as in the storage of calcium ions. SR is built from the formation and maturation of two distinct and functionally related domains: the longitudinal SR and the junctional SR that together wrap the contractile apparatus. T-tubule network is continuous with the muscle cell plasma membrane (PM) and begins from the invagination of PM in a repeated pattern at each sarcomere (Barone et al, 2015). These two-membrane systems are structurally associated to maintain their typical organization in muscle cells. SH3KBP1 depletion seems to alter more specifically junctional SR rather than longitudinal SR as it specifically alters transversal organization of T-tubules (Fig. 6A-C). One hypothesis, recently suggested by Quon et al, is that non-vesicular lipid transport and lipid biosynthesis could intersect at ER-PM membrane contact sites and would serve as a nexus, coordinating requirements in the PM for lipids with their production in the ER (Quon et al, 2018).

Autosomal dominant CNM is caused by heterozygous mutations in the DNM2 gene, which encodes the Dynamin 2 (DNM2) GTPase enzyme (Romero, 2010). DNM2-related autosomal dominant (AD)-CNM was initially 415 characterized as a slowly progressing muscle weakness affecting distal muscles with onset in early adulthood. 
DNM2-R465W missense mutation represents the most frequent mutation in humans and a knock-in (KI) mouse model expressing this mutation has been generated that develops a progressive muscle weakness (Durieux et al, 2010). Expressing DNM2-R465W mutation in mice leads to contractile impairment that precedes muscle atrophy and structural disorganization that mainly affects both mitochondria and endo/sarcoplasmic reticulum. Interestingly, CNM- KI-Dnm2 $2^{\mathrm{R} 465 \mathrm{~W}}$ mouse model exhibits twice more Sh3kbp1 mRNA amount in the first 4 months than control mice before normalization after 8 months (Fig. 7A). Of note, this increase is concomitant with transient transcriptional activation of both ubiquitin-proteasome and autophagy pathways at 2 months of age in the TA muscle of the KI-Dnm2 mice (Durieux et al, 2010). One can hypothesize that this elevated amount of sh $3 \mathrm{kbp} 1$ is one of the factors that limits activation of autophagy pathway activation and thus slows-down CNM associated phenotype. In accordance with this hypothesis, we find that sh3kbp1 depletion increase CNM phenotype (Fig. 7D-H). In conclusion, increased amount of SH3KBP1 could delay the CNM phenotype development by stabilizing Triads and the braking of the autophagy response.

Finally, our data show that Sh3kbp1 depletion increases fusion events in transfected primary myoblasts and stable cell line. In vivo, when Sh3kbp1 is depleted from mature myofibers in a period of time characterized by minimal myonuclei accretion in muscle fibers, we observe a tendency to slightly increase the percentage of myonuclei per myofibers in both WT and KI-Dnm2 $2^{\mathrm{R} 465 \mathrm{~W}}$ conditions (Fig. S3E). These data suggest that SH3KBP1 could contribute to fusion efficiency by a still unknown mechanism. The role of ER on myoblast fusion is poorly documented and its effect seems to be more indirectly linked to pathways related to physiologic ER stress signaling and SARC (stress-activated response to $\mathrm{Ca}^{2+}$ ) body formation more than a direct impact on myoblast fusion (Bohnert et al, 2017; Nakanishi et al, 2007; 2015). Alternatively, our in vitro data suggest that the global microtubule network organization is not changed but that dynamics of organelles related to microtubules pathways are improved, reflected by the increase of myonuclei dynamics in the absence of SH3KBP1 (Fig. 2A-F). There is also no evidence of a clear specific role of microtubules network on the fusion potential, however, the modification of microtubule dynamics/orientation by different MAPs are known to alter fusion potential (Mogessie et al, 2015; Straube \& Merdes, 2007; Cadot et al, 2012). Fusion processes require remodeling of both membranes and actin cytoskeleton polymerization at the site where two membrane will fuse (Sampath et al, 2018). One can hypothesize that SH3KBP1 could contribute to the fusion process through the control of membrane remodeling on specifics PM-ER sites. Incidentally, we noticed that in absence of SH3KBP1, myotubes are longer, increasing consequently the fusiform shape of cells (Fig. 1D). As fusion mainly occurs at the tips of myotubes (Cadot et al, 2015; 2012), this increase of myotubes polarization could contribute to accumulate proteins involved in fusion specifically at the tips of myotubes and consequently favor fusion capacity.

Altogether these data are in agreement with an involvement of Sh3kbp1 in muscle fibers formation and maintenance. In the present study, we show that the adaptor protein SH3KBP1, through its N-terminus part, is able to scaffold ER via an interaction with Calnexin. SH3KBP1 also governs myonuclei motion and spatial organization as well as myoblast fusion. Additionally, we observed that SH3KBP1 interacts with DNM2 and organizes T-tubule formation in skeletal muscle; moreover, its down-regulation contributes to an increase of the CNM-like phenotype in the most frequent mutation model (KI-Dnm2 $2^{\mathrm{R} 465 \mathrm{~W}}$ ) of autosomal dominant centronuclear myopathy. Altogether, our data suggest that SH3KBP1 increase observed in AD-CNM model could be an attempt 
of compensatory mechanism in $\mathrm{CNM}-\mathrm{KI}-\mathrm{Dnm} 2^{\mathrm{R} 465 \mathrm{~W}}$ model and could be used as a preventing factor in the development of CNM phenotype.

\section{Figures}

Figure 1: Myonuclear positioning during primary myotubes formation is controlled by sh3kbp1. (A)

Scheme of sequential steps in order to obtain immature myotubes from primary myoblasts. siRNAs were transfected 24 hours before myoblasts fusion. (B-C) Representative immunofluorescence staining of myosin heavy chain (green) and myonuclei (red) in primary myotubes treated for scramble (B) or a pool of 2 individual siRNAs targeting Sh3kbp1 mRNA (C) after 3 days of differentiation, white arrows indicate "tips-aggregated myonuclei". Scale Bar: $150 \mu \mathrm{m}$. (D-E) Myotubes lengths and Mean distances between each myonuclei and myotube's centroid (DMcM) ranked by myonuclei content per myotubes were quantified after 3 days of differentiation in cells treated with a scramble or Sh3kbp1 siRNAs. Data from three independent experiments were combined. Scramble siRNA cells $(\mathrm{n}=1010)$ and Sh3kbp1 siRNA cells $(\mathrm{n}=1093)$, Unpaired t-test, ${ }^{* * *} \mathrm{p}<0.001$. Center lines show the medians; box limits indicate the 25th and 75th percentiles as determined by R software; whiskers extend 1.5 times the interquartile range from the 25th and 75th percentiles, outliers are represented by dots. (F) Myonuclei Spreading Graph (MSG) represents statistical spatial distribution of myonuclei along myotubes in scramble and in Sh3kbp1 siRNA-treated myotubes; white-line represents the mean value of the statistical frequency.

Figure 2: Myonuclei localization and motion are affected in sh3kbp1 depleted primaries myofibers and

lead to myonuclei aggregation. (A) Scheme of sequential steps in order to obtain mature myofibers from primary mice myoblasts. siRNAs or shRNA were transfected during precocious steps of myoblasts fusion. (B-F) Primary myoblasts were treated with scramble or Sh3kbp1 shRNA tagged with GFP (Green) and co-transfected with RFP-lamin-chromobody ${ }^{\circledR}$ (red) and were induced to differentiate for 5 days into myofibers. (B-D) Quantification of myonuclei clustering by myotubes (B), myonuclei time in motion (C) and myonuclei speed (D) were quantified using SkyPad analysis (Cadot et al, 2014). Data from three independent experiments were combined. Unpaired t-test, ${ }^{* * *} \mathrm{p}<0.001,{ }^{* *} \mathrm{p}<0.01$. (E) Representative immunofluorescence staining of RFPlamin-chromobody ${ }^{\circledR}$ (red) and shRNA tagged with GFP (Green) in primary myotubes treated with scramble or Sh3kbp1 shRNA after 5 days of differentiation into myofibers. Scale Bar: $150 \mu \mathrm{m}$. (Asterisks represents myonuclei clustering) (F) Frames from a 16h time-lapse movie in two channels (shRNA in green and lamin-chromobody® in red) of primary myotubes. In the first frame (on the left), myofibers are selected in white, which corresponds

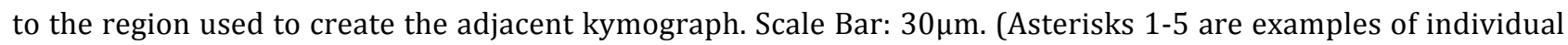
myonuclei tracking). (G) Four representative images of 10 days differentiated myofibers transfected with either


individual myonuclei) (H-I) Quantification of the mean distance between pairwise myonuclei $(\mathrm{H})$ and mean myofibers width (I) in 10 days differentiated myofibers treated with scramble siRNA or shRNA, a pool of 2 individual siRNAs or an individual shRNAs targeting Sh3kbp1. Data from three independent experiments were 
software; whiskers extend 1.5 times the interquartile range from the 25th and 75 th percentiles, outliers are represented by dots. Unpaired t-test, ${ }^{* * *} \mathrm{p}<0.001,{ }^{* *} \mathrm{p}<0.01,{ }^{*} \mathrm{p}<0.05$.

Figure 3: SH3KBP1 is induced during myotubes differentiation and controls myonuclei aggregation in

C2C12 cells. (A) Western blot analysis of SH3KBP1 protein expression in total protein extracts from proliferating (P) or differentiating C2C12 cells for up to 5 days. C2C12 differentiation is assessed by Myogenin expression and Tubulin is used as loading control. (B-C) qRT-PCR analysis of Sh3kbp1 (B) and Myogenin (C) gene expression level relative to CycloB, Gapdh, GusB, Rpl41 and Tbp gene in proliferating C2C12 cells (P) or differentiating C2C12 cells for up to 5 days. (D) Representative western blots analysis of SH3KBP1 protein downregulation in stable cell line constitutively expressing shRNA construct targeting Sh3kbp1. Tubulin is used as loading control. (E) Representative immunofluorescence staining of myosin heavy chain (green) and myonuclei (red) in 3 or 6 days cultured C2C12 stable cell lines expressing scramble shRNA or shRNA targeting Sh3kbp1. Zooms $1 \& 2$ are magnifications of images in white dots rectangle in 6 days old myotubes. Scale bars, $150 \mu \mathrm{m}$ and $15 \mu \mathrm{m}$ in zoom. (F) Quantification of the number of myonuclei by clusters in C2C12 cells stable cell line expressing scramble shRNA or shRNA targeting Sh3kbp1 after 5 days of differentiation. Data from three independent experiments were combined. Unpaired t-test, ${ }^{* * *} \mathrm{p}<0.001,{ }^{*} \mathrm{p}<0.05$. Center lines show the medians; box limits indicate the 25th and 75th percentiles as determined by R software; whiskers extend 1.5 times the interquartile range from the 25 th and 75 th percentiles, outliers are represented by dots.

512 Figure 4: SH3KBP1 is localized near and inside myonuclei and controls ERP72 positives Endoplasmic reticulum through Calnexin interaction during in vitro muscle fibers differentiation. (A-B) Representative immunofluorescent staining of SH3KBP1 (green), Actin (Red) and myonuclei (blue) in the course of myotube formation in C2C12 cells line in proliferation (P) or after 2 and 5 days of differentiation (A) or in the course of myofibers formation in primary myoblast cultures in proliferation (P) or after 3 and 10 days of differentiation (B). Scale bars, $10 \mu \mathrm{m}$. (C-D) Representative Immunofluorescent staining of Golgi Marker (RCAS1, for receptor binding cancer antigen expressed on SiSo cells, red) (C) and Endoplasmic Reticulum (ERP72, for Endoplasmic reticulum resident protein 72, red) (D) and myonuclei (blue) in 6 days cultured C2C12 stable cell line expressing either scramble shRNA or shRNA targeting Sh3kbp1 gene (GFP-shRNA). Scale bars, $100 \mu \mathrm{m}$. (E) Scheme of SH3KBP1 constructs, labeled with GFP in the N-terminus part, used for immunoprecipitation assay in (F-G). FL: full length. (F) Representative western blot with indicated antibodies (right) in C2C12s expressing GFPSH3KBP1 constructs (top). (G) Representative western blot with indicated antibodies (right) of GFP-SH3KBP1 constructs immunoprecipitation using $\mathrm{C} 2 \mathrm{C} 12$ expressing indicated constructs (top). Blots were repeated more than 3 times.

Figure 5: SH3KBP1 is localized near myonuclei and at I-band/Z-line zone in muscle fibers in vivo and interact with DNM2. (A-B) Representative images of Tibialis Anterior muscle transversals cross-section stained for SH3KBP1 (green) and myonuclei (red). Asterisks show myonuclei inside myofibers. Scale bars, $150 \mu \mathrm{m}$. (C-E) Representative images of Tibialis Anterior muscle longitudinal cross-section stained for SH3KBP1 (red) and DHPR1 $\alpha$, for DyHydroPyridine Receptor alpha (green). Scale bars, $150 \mu \mathrm{m}$. (F) Representative immunofluorescent staining of long term expression of SH3KBP1 constructs (listed in Fig. 4E) in green (GFPconstructs), Actin (red) and myonuclei (blue) in 10 days differentiated primary myofibers. Scale bars, $10 \mu \mathrm{m} .(\mathrm{G})$ 
Line scan of the yellow boxes to visualize transversal organization of SH3KBP1 (green) and actin (red) staining. (H) Western blot with indicated antibodies (right) in C2C12 cells expressing Flag-SH3KBP1 constructs (top) and GFP-DNM2. (I) Representative western blot with indicated antibodies (right) of DNM2-GFP constructs immunoprecipitation using C2C12 expressing indicated constructs (top).

Figure 6: SH3KBP1 controls T-tubule organization during muscle fibers formation and in mature myofibers. (A-B) Representative immunofluorescent image of DHPR1- $\alpha$ (green), Actin (red) and myonuclei (blue) staining in 10 days cultured primary myotubes after scramble siRNA (A) or Sh3kbp1 siRNA (B) transfection. Scale bars, $20 \mu \mathrm{m}$. (C) Quantification of DHPR- $\alpha$ staining aspects in mature myofibers treated with either scramble siRNA, a pool of 2 individual siRNAs targeting Sh3kbp1 mRNA, scramble shRNA or an individual shRNA targeting Sh3kbp1 mRNA. Data from three independent experiments were combined. Unpaired t-test, ${ }^{* * *} \mathrm{p}<0.001,{ }^{* *} \mathrm{p}<0.01$. Details for different categories are available in Fig. S2E. (D-E) Representative images of transversals (left) cross-section of Tibialis Anterior muscle electroporated with scramble or an individual shRNAs targeting Sh3kbp1 and stained for GFP (green) and myonuclei (blue). Scale bars, $50 \mu \mathrm{m}$ (F) Quantification of muscle fibers electroporated with scramble or an individual shRNAs targeting $\operatorname{Sh} 3 k b p 1$. Center lines show the medians; box limits indicate the 25th and 75th percentiles as determined by R software; whiskers extend 1.5 times the interquartile range from the 25th and 75th percentiles, outliers are represented by dots. Unpaired t-test, ${ }^{* * *} \mathrm{p}<0.001$. (G-H) Representative images of longitudinal cross-section of Tibialis Anterior muscle electroporated with scramble or an individual shRNAs targeting Sh3kbp1 and stained for GFP and DHPR$\alpha$. Scale bars, $20 \mu \mathrm{m}$.

Figure 7: sh3kbp1 silencing worsened CNM phenotype in KI-DNM2 ${ }^{\text {R465W }}$ mice model. (A) qRT-PCR analysis of Sh3kbp1 gene expression relative to Nat10 gene Tibialis Anterior muscles from WT or KI-Dnm2 ${ }^{\mathrm{R} 465 \mathrm{~W}}$ mice at 1 , 2, 4 and 8 months of age. Non-parametric one-way Anova used for statistical significance ${ }^{* * *} p<0.001,{ }^{* *} p<0.01,{ }^{*} p$ $<0.05$. (B-C) Representative images of Tibialis Anterior muscle cross-section from WT or KI- Dnm2 $2^{\mathrm{R} 465 \mathrm{~W}} \mathrm{mice}$ at the age of 4 months, injected with either PBS or AAV cognate vector expressing shRNA targeting SH3KBP1 mRNA (Sh3kbp1-shRNA) for 3 months and stained for Dapi (Blue), Laminin (green) and MyHC (red). Scale Bars = 150 $\mu \mathrm{m}$. (D-H) Quantification of Absolute force (P0) (D), Tibialis Anterior muscle mass (E), mean cross-sectioned myofibers areas (F), percentage of slow fibers (MHC1 positives) (G) and percentage of LC3II-positive fibers (H) of Tibialis Anterior muscles from WT or KI- Dnm2 $2^{\mathrm{R} 465 \mathrm{~W}}$ mice at the age of 4 months, injected with either PBS or AAV cognate vector expressing shRNA targeting SH3KBP1 mRNA (Sh3kbp1-shRNA) for 3 months. Data from at least 4 mice in each condition were combined. Unpaired t-test, ${ }^{* * *} \mathrm{p}<0.001,{ }^{* *} \mathrm{p}<0.01,{ }^{*} \mathrm{p}<0.05$. Center lines show the medians; box limits indicate the 25th and 75th percentiles as determined by R software; whiskers extend 1.5 times the interquartile range from the 25 th and 75 th percentiles, outliers are represented by dots.

Supplementary figure 1: SH3KBP1 affects myoblast fusion. (A) Representative western blot analysis of SH3KBP1 protein expression in total protein extracts from differentiating primary myotubes treated with scramble or siRNA (\#1 \& \#2) or a pool of siRNA targeting SH3KBP1 mRNA. Loading control is tubulin. (B-C)

572 Representative immunofluorescent images of 3 days of primary differentiated myotubes stained for myosin 


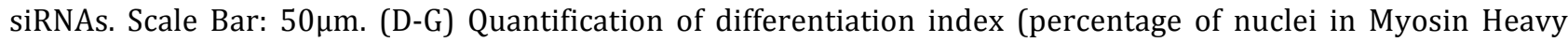
Chain positives cells) (D), Fusion index (percentage of nuclei inside myotubes) (E), Average number of nuclei by myotubes (F) and distribution of myotubes classified depending on the number of nuclei in myotubes (G). Data from three independent experiments were combined. Unpaired t-test, ${ }^{* *} \mathrm{p}<0.01,{ }^{*} \mathrm{p}<0.05$. Center lines show the medians; box limits indicate the 25th and 75th percentiles as determined by R software; whiskers extend 1.5 times the interquartile range from the 25 th and 75 th percentiles, outliers are represented by dots.

\section{Supplementary figure 2: SH3KBP1 affects myoblast fusion and localization of myonuclei in C2C12 cells.} (A-B) Distribution of 3 days differentiated C2C12 myotubes formed from stable cell line expressing either scramble or Sh3kbp1 shRNAs and classified depending on nuclei content inside myotubes after (A) 3 days or (B) 5 days of differentiation. (C) Representatives immunofluorescent staining of myosin heavy chain (green) and myonuclei (red) in stable cell line expressing Sh3kbp1 shRNA (top) or co-transfected with full-length SH3KBP1 (bottom) after 5 days of differentiation. Scale Bar: $50 \mu \mathrm{m}$. (D) Quantification of the percentage of nuclei by clusters in stable cell line expressing Sh3kbp1 shRNA and co-transfected with mCherry or full-length SH3KBP1 plasmid. Data from three independent experiments were combined. Unpaired t-test, ${ }^{* * *} p<0.001,{ }^{* *} p<0.01,{ }^{*} p$ $<0.05$. Center lines show the medians; box limits indicate the 25 th and 75 th percentiles as determined by $\mathrm{R}$ software; whiskers extend 1.5 times the interquartile range from the 25th and 75th percentiles, outliers are represented by dots. (E) Representatives immunofluorescent categories of staining for DHPR-alpha in 10 days differentiated myofibers. Scale Bar: $2 \mu \mathrm{m}$.

Supplementary figure 3: sh3kbp1 silencing worsened CNM phenotype in KI-DNM2 ${ }^{\mathrm{R} 465 \mathrm{~W}}$ mice model. (A) qRT-PCR analysis of Sh3kbp1 gene expression relative to Nat10 gene in 4 months Tibialis Anterior muscles from WT or KI- Dnm2 $2^{\mathrm{R} 465 \mathrm{~W}}$ mice injected with either PBS or AAV cognate vector expressing shRNA targeting SH3KBP1 mRNA (Sh3kbp1-shRNA). ${ }^{* * *}$ p $<0.001$. (B-F) Quantification of total body mass (B), distribution of crosssectioned myofibers areas (C), number of fibers by muscles (D), number of myonuclei by fiber (E) and number of internalized myonuclei (F) in Tibialis Anterior muscles from WT or KI-Dnm2 $2^{\mathrm{R} 465 \mathrm{~W}}$ mice at the age of 4 months, injected with either PBS or AAV cognate vector expressing shRNA targeting SH3KBP1 mRNA (AAV-SH3KBP1) for 3 months. Data from at least 4 mice in each condition were combined. Unpaired t-test, ${ }^{* * *} \mathrm{p}<0.001,{ }^{* *} \mathrm{p}<0.01$, ${ }^{*} \mathrm{p}<0.05$. Center lines show the medians; box limits indicate the 25 th and 75 th percentiles as determined by $\mathrm{R}$ software; whiskers extend 1.5 times the interquartile range from the 25th and 75th percentiles, outliers are represented by dots. (G) Immunofluorescent staining of SH3KBP1, Actin and nuclei in two control human patient and in two patients expressing DNM2-R465W point mutation.

Supplementary video 1 \& 2: Timelapse experiments of primary myoblasts co-transfected with LaminChromobody ${ }^{\circledR}$-RFP plasmids and with either Scramble-siRNA (Supplementary video-1) or Sh3kbp1-siRNA 


\section{Cell culture}

615 Primary myoblasts were collected from wild type C57BL6 mice as described before (Falcone et al, 2014; Pimentel et $616 a l$, 2017). Briefly, Hindlimb muscles from 6 days pups were extracted and digested with collagenase (Sigma, C9263$6171 \mathrm{G}$ ) and dispase (Roche, 04942078001). After a pre-plating step to discard contaminant cells such as fibroblasts, 618 myoblasts were cultured on matrigel coated-dish (Corning, 356231) and induced to differentiate in myotubes for 2-3 619 days in differentiation media (DM: IMDM (Gibco, 21980-032) $+2 \%$ of horse serum (Gibco, 16050-122) $+1 \%$ 620 penicillin-streptomycin (Gibco, 15140-122)). Myotubes were then covered by a concentrated layer of matrigel and 621 maintained for up to 10 days in long differentiation culture medium (LDM: IMDM (Gibco, 21980-032) + 2\% of horse serum (Gibco, 16050-122) + 0.1\% Agrin $+1 \%$ penicillin-streptomycin (Gibco, 15140-122)) until the formation of mature and contracting myofibers. LDM was changed every two days.

Mouse myoblast C2C12 cells were cultured in Dulbecco's modified Eagle's medium (DMEM (Gibco, 41966029) + 15\% fetal bovine serum (FBS) (Gibco, 10270-106) + 1\% penicillin-streptomycin (Gibco, 15140-122))) and were plated on $0.1 \%$ matrigel-coated dishes for 1-2 days before differentiation. Differentiation was induced by switching to differentiation media (DMEM $+1 \%$ horse serum).

\section{Cell transfection}

For $\mathrm{C} 2 \mathrm{C} 12$ cells, 3 different siRNAs Silencer per gene were transfected using Lipofectamine 2000 (ThermoFisher Scientifics, 11668-019) at the final concentration of $10 \mathrm{nM}$, following manufacturer instructions, 2 days before differentiation. For shRNA cDNA (Geneocopia) transfection, Lipofectamine 2000 (ThermoFisher Scientifics, 11668019) was used following manufacturer instructions. For primary cells, siRNA were transfected using Lipofectamine 2000 (ThermoFisher Scientifics, 11668-019) at the final concentration of $2 \mathrm{nM}$. shRNA (Geneocopia), Eb1 or RFP-Lamin-chromobody (Chromotek) cDNA were transfected using Lipofectamine 3000 (ThermoFisher Scientifics, L3000-008). For the list of siRNA, shRNA and DNA constructs, refer to supplementary table 1 .

\section{Protein sample preparation}

For primary cultured cells or $\mathrm{C} 2 \mathrm{C} 12$ cell lines, cells were harvested, using Trypsin for $5 \mathrm{~min}$ at $37^{\circ} \mathrm{C}$ and centrifuged at 1500RPM for $5 \mathrm{~min}$ at $4^{\circ} \mathrm{C}$. Cell pellets were diluted and incubated in the optimal volume of RIPA lysis buffer containing phosphatases inhibitors (Sigma, P5726-5mL) and proteases inhibitors (Sigma, P8340) for $10 \mathrm{~min}$ at $4^{\circ} \mathrm{C}$. Following a sonication and a centrifugation at $12000 \mathrm{RPM}$ for $10 \mathrm{~min}$ at $4^{\circ} \mathrm{C}$, protein samples were collected for further uses. The concentration of proteins was determined using BCA protein assay kit (Thermo Fisher Scientifics, 23225) as described by the manufacturer.

\section{Western blot}

To carry out western blots, the same amount of sample were loaded in $6 \%$ acrylamide gels and were migrated at $130 \mathrm{~V}$ for $10 \mathrm{~min}$ followed by $160 \mathrm{~V}$ for $90 \mathrm{~min}$. iBlot 2 mini slacks (Thermo Fisher Scientifics, IB23002) semi-dry system was used to transfer the proteins to nitrocellulose membranes. Membranes were then saturated in $5 \%$ milk in TBS for $1 \mathrm{~h}$ at 
room temperature (RT) and were incubated in primary antibodies in $5 \%$ milk in TBS over night at $4{ }^{\circ} \mathrm{C}$. Following washes by $0.1 \%$ Tween-20-1X TBS, the membranes were incubated in HRP conjugated secondary antibodies in $5 \%$ milk in TBST for $1 \mathrm{~h}$ at room temperature (RT). Following washes by $0.1 \%$ Tween-20-1X TBS the detection of the target proteins was carried out using Super Signal West Femto (Thermo Fisher Scientifics, 34095) and ChemiDoc imaging system (BioRad).

\section{Antibodies}

Cells were fixed in $4 \% \mathrm{PFA}$ in PBS for $20 \mathrm{~min}$ at $37^{\circ} \mathrm{C}$ followed by washes with PBS and permeabilization with $0.5 \%$ Triton-X100 in PBS for 5min at RT. Following washes with PBS, cells were saturated with 1\% BSA in PBS for 30min at $37^{\circ} \mathrm{C}$ and incubated in primary antibodies over night at $4^{\circ} \mathrm{C}$. Following washes with $0.05 \%$ Triton-X100 in PBS, cells were incubated in secondary antibodies or dyes for $2 \mathrm{hrs}$ at RT followed by washes with $0.05 \%$ Triton-X100 in PBS and a last wash in PBS. Cultured myofibers were imaged using either Z1-AxioObserver (Zeiss) or confocal SP5 microscope (Leica). For the list of antibodies and dilution, refer to supplementary table 2.

\section{Video-Microscopy}

665 Time-lapse images were acquired using Z1-AxioObserver (Zeiss) with intervals of 20 minutes. Final videos were analyzed using Metamorph (Zeiss) and SkyPad plugin as described before (Cadot et al, 2014).

\section{Adeno-Associated Virus production and in vivo transduction}

A cassette containing the small hairpin (sh) RNA under the control of H1 RNA polymerase III promoter was inserted in a pSMD2 expression plasmid. AAV vectors (serotype 1) were produced in HEK293 cells after transfection of the pSMD2-shRNA plasmid, the pXX6 plasmid coding for viral helper genes essential for AAV production and the pRepCap plasmid (p0001) coding for AAV1 capsid as described previously (Riviere et al, 2006). Viral particles were purified on iodixanol gradients and concentrated on Amicon Ultra-15 100K columns (Merck-Millipore). The concentration of viral genomes $(\mathrm{vg} / \mathrm{ml})$ was determined by quantitative real-time PCR on a LightCycler480 (Roche diagnostic, France) by using TaqMan probe. A control pSMD2 plasmid was tenfold serially diluted (from $10^{7}$ to $10^{1}$ copies) and used as a control to establish the standard curve for absolute quantification. Male wild type and heterozygous $\mathrm{KI}-\mathrm{Dnm} 2^{\mathrm{R} 465 \mathrm{~W}}$ mice were injected under isoflurane anesthesia. Two intramuscular injections of $30 \mu 1$ within $24 \mathrm{~h}$ interval were performed using $29 \mathrm{G}$ needle in TA muscles corresponding to $10^{11}$ viral genomes per muscle. All of the experiments and procedures were conducted in accordance with the guidelines of the local animal ethics committee of the University Claude Bernard - Lyon 1 and in accordance with French and European legislation on animal experimentation and approved by the ethics committee CECCAPP and the French ministry of research.

\section{Muscle contractile properties}

684 The isometric contractile properties of TA muscles were studied in situ on mice anesthetized with $60 \mathrm{mg} / \mathrm{kg}$ pentobarbital. The distal tendon of the TA muscle was attached to a lever arm of a servomotor system (305B Dual- 
Mode Lever, Aurora Scientific). The sciatic nerve was stimulated by a bipolar silver electrode using a supramaximal $(10 \mathrm{~V})$ square wave pulse of $0.1 \mathrm{~ms}$ duration. Absolute maximal isometric tetanic force was measured during isometric contractions in response to electrical stimulation (frequency of $25-150 \mathrm{~Hz}$; train of stimulation of $500 \mathrm{~ms}$ ). All isometric contraction measurements were made at optimal muscle length. Force are expressed in grams $(1 \mathrm{gram}=9.8$ mNewton). Mice were sacrificed by cervical dislocation and TA muscles were weighted. Specific maximal force was calculated by dividing absolute force by muscle weight.

\section{RNA extraction}

693 After the addition of Trizol (Sigma, T9424-200mL) on each sample, lysing matrix D and fast prep system (MPbio, 694 6913-100) were used for sample digestion and pre-RNA extraction. In order to extract RNA, samples were incubated in 695 chloroform for $5 \mathrm{~min}$ at RT, centrifuged for $15 \mathrm{~min}$ at $12000 \mathrm{rcf}$ at $4^{\circ} \mathrm{C}$ and incubated in the tubes containing isopropanol 696 (precipitatation of RNA) for $10 \mathrm{~min}$ at RT. following a centrifuge of samples for $15 \mathrm{~min}$ at $12000 \mathrm{rcf}$ at $4^{\circ} \mathrm{C}$, samples were washed 2 times with $70 \%$ ethanol and the final RNA pellets were diluted in ultra-pure RNase free water (Invitrogen, 10977-035). RNA concentration was calculated using Nanodrop (ThermoFisher Scientifics).

\section{RT-q-PCR on cells}

Goscript Reverse Transcriptase System (Promega, A5001) was used, as described by the manufacturer to produce the cDNA. Fast Start Universal SYBR Green Master (Rox)(Roche, 04913914001) and CFX Connect ${ }^{\mathrm{TM}}$ Real-Time PCR Detection System (BioRad) were used to carry out the quantitative PCR using the following primer sets. The CT of target genes were normalized on 3 control genes. For the list of primers used, refer to supplementary table 1.

\section{RT-q-PCR on muscle samples}

50 longitudinal sections $(12 \mu \mathrm{m})$ of TA muscles were cut and used for RNA isolation and RT-qPCR. Total RNA was extracted from muscle by using NucleoSpin (Macherey-Nagel). RNA (200 ng) was reverse transcribed using Reverse Transcription Core Kit (Eurogentec). Real-time PCR was performed in a $20 \mu \mathrm{L}$ final volume using the Takyon No Rox SYBR kit (Eurogentec). Fluorescence intensity was recorded using a CFX96 Real-Time PCR Detection System (Bio$\mathrm{Rad}$ ) and the data analyzed using the $\Delta \Delta \mathrm{Ct}$ method of analysis. Reference gene $18 \mathrm{~s}$ was used to normalize the expression level of the gene of interest as previously described (Pfaffl et al., 2001). The selected forward and reverse primer sequences are listed in Table 1. Statistical analyses were performed using GraphPad PRISM 5.0 (La Jolla). Data were analyzed for normal distribution using Shapiro-Wilk test. Non-parametric one-way Anova $(n=4-6)$ was used to determine transcripts expression level. Primers were designed using Primer 3 software from gene sequences obtained from Genebank. Primer specificity was determined using a BLAST search. For the list of primers used, refer to supplementary table 1.

\section{Histological staining and analysis}

Tibialis anterior muscles were collected, embedded in tragacanth gum, and quickly frozen in isopentane cooled in 
histological, immunohistochemical, enzymohistochemical analyses according to standard protocols. The fibre crosssectional area and the number of centrally nucleated fibers were determined using Laminin and Dapi-stained sections. Fluorescence microscopy and transmission microscopy were performed using Axioimager Z1 microscope with CP Achromat $5 \mathrm{x} / 0.12,10 \mathrm{x} / 0.3 \mathrm{Ph} 1$, or 20x/0.5 Plan NeoFluar objectives (Zeiss). Images were captured using a chargecoupled device monochrome camera (Coolsnap HQ, Photometrics) or color camera (Coolsnap colour) and MetaMorph software. For all imaging, exposure settings were identical between compared samples. Fiber number and size, central nuclei and peripheral myonuclei were calculated using ImageJ software.

\section{Quantification methods for myonuclei spreading in myotubes}

Quantifications in immature myotubes were assessed using an homemade analysis tool. An image analysis performed in Image ${ }^{\circledR}$ software is combined with a statistical analysis in RStudio ${ }^{\circledR}$ software. This provides quantifications of parameters, ranked by myonuclei content per myotubes, regarding phenotype of myotubes (area, length) and their respective myonuclei positioning compare to centroid of myotubes (DMcM).

MSG diagrams were obtained through the normalization of lengths of all analyzed myotubes (independently to their myonuclei content) to $100 \%$. White lines represent myonuclei density curves assessing the statistical frequency for myonuclei positioning along myotubes. Each color group reflects statistical estimation of myonuclei clustering along myotubes.

\section{Additional information}

\section{Competing interests}

740 The authors declare no competing interests.

\section{Funding}

742 This work was funded by grants from ATIP-AVENIR Program and Association Française contre les Myopathies (MyoNeurAlp Alliance).

Acknowledgements

We thank the Penn Vector Core, Gene Therapy Program (University of Pennsylvania, Philadelphia, US) for providing pAAV1 plasmid (p0001), and Sofia Benkhelifa-Ziyyat for AAV production, the Imaging facilities of Lyon, PLATIM and CICLE, the animal facility of Lyon, PBES, and particularly, Christophe Chamot and Claire Burny for building the macros in ImageJ and R-Studio.

\section{Author contributions}

Conceptualization, A.G., M.B. and V.G.; Methodology, A.G., N.C., M.B., A.G., A-C.D.and V.G. Formal Analysis,

\section{References}


bioRxiv preprint doi: https://doi.org/10.1101/2020.05.04.076208; this version posted May 5, 2020. The copyright holder for this preprint (which was not certified by peer review) is the author/funder. All rights reserved. No reuse allowed without permission.

Al-Qusairi L \& Laporte J (2011) T-tubule biogenesis and triad formation in skeletal muscle and implication in human diseases. Skelet Muscle 1: 26

Al-Qusairi L, Weiss N, Toussaint A, Berbey C, Messaddeq N, Kretz C, Sanoudou D, Beggs AH, Allard B, Mandel J-L, Laporte J, Jacquemond V \& Buj-Bello A (2009) T-tubule disorganization and defective excitation-contraction coupling in muscle fibers lacking myotubularin lipid phosphatase. Proc. Natl. Acad. Sci. U.S.A. 106: 1876318768

Barone V, Randazzo D, Re V, Sorrentino V \& Rossi D (2015) Organization of junctional sarcoplasmic reticulum proteins in skeletal muscle fibers. Journal of muscle research and cell motility 36: 501-515

Bevilacqua JA, Monnier N, Bitoun M, Eymard B, Ferreiro A, Monges S, Lubieniecki F, Taratuto AL, Laquerrière A, Claeys KG, Marty I, Fardeau M, Guicheney P, Lunardi J \& Romero NB (2011) Recessive RYR1 mutations cause unusual congenital myopathy with prominent nuclear internalization and large areas of myofibrillar disorganization. Neuropathol. Appl. Neurobiol. 37: 271-284

Bitoun M, Maugenre S, Jeannet P-Y, Lacène E, Ferrer X, Laforêt P, Martin J-J, Laporte J, Lochmüller H, Beggs AH, Fardeau M, Eymard B, Romero NB \& Guicheney P (2005) Mutations in dynamin 2 cause dominant centronuclear myopathy. Nature genetics 37: 1207-1209

Blondelle J, Ohno Y, Gache V, Guyot S, Storck S, Blanchard-Gutton N, Barthélémy I, Walmsley G, Rahier A, Gadin S, Maurer M, Guillaud L, Prola A, Ferry A, Aubin-Houzelstein G, Demarquoy J, Relaix F, Piercy RJ, Blot S, Kihara A, et al (2015) HACD1, a regulator of membrane composition and fluidity, promotes myoblast fusion and skeletal muscle growth. J Mol Cell Biol 7: 429-440

Bohnert KR, McMillan JD \& Kumar A (2017) Emerging roles of ER stress and unfolded protein response pathways in skeletal muscle health and disease. J. Cell. Physiol 233: 67-78

Bruusgaard JC, Liestøl K \& Gundersen K (2006) Distribution of myonuclei and microtubules in live muscle fibers of young, middle-aged, and old mice. Journal of Applied Physiology 100: 2024-2030

Bruusgaard JC, Liestøl K, Ekmark M, Kollstad K \& Gundersen K (2003) Number and spatial distribution of nuclei in the muscle fibres of normal mice studied in vivo. The Journal of Physiology 551: 467-478

Buchman VL, Luke C, Borthwick EB, Gout I \& Ninkina N (2002) Organization of the mouse Ruk locus and expression of isoforms in mouse tissues. Gene 295: 13-17

Burgoyne T, Morris EP \& Luther PK (2015) Three-Dimensional Structure of Vertebrate Muscle Z-Band: The Small-Square Lattice Z-Band in Rat Cardiac Muscle. Journal of molecular biology 427: 3527-3537

Cadot B, Gache V \& Gomes ER (2014) Fast, multi-dimensional and simultaneous kymograph-like particle dynamics (SkyPad) analysis. PLoS ONE 9: e89073

Cadot B, Gache V \& Gomes ER (2015) Moving and positioning the nucleus in skeletal muscle - one step at a time. Nucleus 6: 373-381

Cadot B, Gache V, Vasyutina E, Falcone S, Birchmeier C \& Gomes ER (2012) Nuclear movement during myotube formation is microtubule and dynein dependent and is regulated by Cdc42, Par6 and Par3. EMBO Rep. 13: 741-749

Chin Y-H, Lee A, Kan H-W, Laiman J, Chuang M-C, Hsieh S-T \& Liu Y-W (2015) Dynamin-2 mutations associated with centronuclear myopathy are hypermorphic and lead to T-tubule fragmentation. Hum. Mol. Genet. 24: $5542-5554$

Doñate Puertas R, Millat G, Ernens I, Gache V, Chauveau S, Morel E, Christin E, Couturier N, Devaux Y \& Chevalier $P$ (2018) Atrial Structural Remodeling Gene Variants in Patients with Atrial Fibrillation. Biomed Res Int 2018: $4862480-12$

Durieux A-C, Vignaud A, Prudhon B, Viou MT, Beuvin M, Vassilopoulos S, Fraysse B, Ferry A, Lainé J, Romero NB, 
bioRxiv preprint doi: https://doi.org/10.1101/2020.05.04.076208; this version posted May 5, 2020. The copyright holder for this preprint (which was not certified by peer review) is the author/funder. All rights reserved. No reuse allowed without permission.

Guicheney P \& Bitoun M (2010) A centronuclear myopathy-dynamin 2 mutation impairs skeletal muscle structure and function in mice. Hum. Mol. Genet. 19: 4820-4836

Falcone S, Roman W, Hnia K, Gache V, Didier N, Laine J, Aurade F, Marty I, Nishino I, Charlet-Berguerand N, Romero NB, Marazzi G, Sassoon D, Laporte J \& Gomes ER (2014) N-WASP is required for Amphiphysin2/BIN1-dependent nuclear positioning and triad organization in skeletal muscle and is involved in the pathophysiology of centronuclear myopathy. EMBO Mol Med 6: 1455-1475

Fongy A, Falcone S, Lainé J, Prudhon B, Martins-Bach A \& Bitoun M (2019) Nuclear defects in skeletal muscle from a Dynamin 2-linked centronuclear myopathy mouse model. Scientific Reports 9: 1580

Fujita N, Huang W, Lin T-H, Groulx J-F, Jean S, Nguyen J, Kuchitsu Y, Koyama-Honda I, Mizushima N, Fukuda M \& Kiger AA (2017) Genetic screen in Drosophila muscle identifies autophagy-mediated T-tubule remodeling and a Rab2 role in autophagy. Elife 6: e23367

Gache V, Gomes ER \& Cadot B (2017) Microtubule motors involved in nuclear movement during skeletal muscle differentiation. Mol. Biol. Cell: mbc.E16-06-0405

Ghasemizadeh A, Christin E, Guiraud A, Couturier N, Risson V, Girard E, Jagla C, Soler C, Laddada L, Sanchez C, Jaque F, Garcia A, Lanfranchi M, Jacquemond V, Gondin J, Courchet J, Schaeffer L \& Gache V (2019) Skeletal muscle MACF1 maintains myonuclei and mitochondria localization through microtubules to control muscle functionalities. bioRxiv 12: 636464

Gimpel P, Lee YL, Sobota RM, Calvi A, Koullourou V, Patel R, Mamchaoui K, Nedelec F, Shackleton S, Schmoranzer J, Burke B, Cadot B \& Gomes ER (2017) Nesprin-1 $\alpha$-Dependent Microtubule Nucleation from the Nuclear Envelope via Akap450 Is Necessary for Nuclear Positioning in Muscle Cells. Current biology: CB

Grumati P, Dikic I \& Stolz A (2018) ER-phagy at a glance. J. Cell. Sci. 131: jcs217364

Gundersen K (2016) Muscle memory and a new cellular model for muscle atrophy and hypertrophy. Journal of Experimental Biology 219: 235-242

Havrylov S, Ichioka F, Powell K, Borthwick EB, Baranska J, Maki M \& Buchman VL (2008) Adaptor protein Ruk/CIN85 is associated with a subset of COPI-coated membranes of the Golgi complex. Traffic 9: 798-812

Havrylov S, Jolanta Redowicz M \& Buchman VL (2010) Emerging Roles of Ruk/CIN85 in Vesicle-Mediated Transport, Adhesion, Migration and Malignancy. Traffic 11: 721-731

Havrylov S, Rzhepetskyy Y, Malinowska A, Drobot L \& Redowicz MJ (2009) Proteins recruited by SH3 domains of Ruk/CIN85 adaptor identified by LC-MS/MS. Proteome Sci 7: 21

Janin A \& Gache V (2018) Nesprins and Lamins in Health and Diseases of Cardiac and Skeletal Muscles. Front Physiol 9: 97

Jungbluth H \& Gautel M (2014) Pathogenic mechanisms in centronuclear myopathies. Front Aging Neurosci 6: 339

Jungbluth H, Zhou H, Sewry CA, Robb S, Treves S, Bitoun M, Guicheney P, Buj-Bello A, Bönnemann C \& Muntoni F (2007) Centronuclear myopathy due to a de novo dominant mutation in the skeletal muscle ryanodine receptor (RYR1) gene. Neuromuscular Disorders 17: 338-345

Kim JH, Jin P, Duan R \& Chen EH (2015) Mechanisms of myoblast fusion during muscle development. Curr. Opin. Genet. Dev. 32: 162-170

Kimura K, Mamane A, Sasaki T, Sato K, Takagi J, Niwayama R, Hufnagel L, Shimamoto Y, Joanny J-F, Uchida S \& Kimura A (2017) Endoplasmic-reticulum-mediated microtubule alignment governs cytoplasmic streaming. Nat. Cell Biol. 19: 399-406

Laporte J, Hu LJ, Kretz C, Mandel JL, Kioschis P, Coy JF, Klauck SM, Poustka A \& Dahl N (1996) A gene mutated in 
bioRxiv preprint doi: https://doi.org/10.1101/2020.05.04.076208; this version posted May 5, 2020. The copyright holder for this preprint (which was not certified by peer review) is the author/funder. All rights reserved. No reuse allowed without permission.

X-linked myotubular myopathy defines a new putative tyrosine phosphatase family conserved in yeast. Nature genetics 13: 175-182

Liu J-X, Höglund A-S, Karlsson P, Lindblad J, Qaisar R, Aare S, Bengtsson E \& Larsson L (2009) Myonuclear domain size and myosin isoform expression in muscle fibres from mammals representing a 100,000-fold difference in body size. Experimental physiology 94: 117-129

Louis M, Zanou N, Van Schoor M \& Gailly P (2008) TRPC1 regulates skeletal myoblast migration and differentiation. J. Cell. Sci. 121: 3951-3959

Metzger T, Gache V, Xu M, Cadot B, Folker ES, Richardson BE, Gomes ER \& Baylies MK (2012) MAP and kinesindependent nuclear positioning is required for skeletal muscle function. Nature 484: 120-124

Mogessie B, Roth D, Rahil Z \& Straube A (2015) A novel isoform of MAP4 organises the paraxial microtubule array required for muscle cell differentiation. Elife 4: e05697

Nakanishi K, Dohmae N \& Morishima N (2007) Endoplasmic reticulum stress increases myofiber formation in vitro. The FASEB Journal 21: 2994-3003

Nakanishi K, Kakiguchi K, Yonemura S, Nakano A \& Morishima N (2015) Transient Ca 2+depletion from the endoplasmic reticulum is critical for skeletal myoblast differentiation. The FASEB Journal 29: 2137-2149

Nicot A-S, Toussaint A, Tosch V, Kretz C, Wallgren-Pettersson C, Iwarsson E, Kingston H, Garnier J-M, Biancalana V, Oldfors A, Mandel J-L \& Laporte J (2007) Mutations in amphiphysin 2 (BIN1) disrupt interaction with dynamin 2 and cause autosomal recessive centronuclear myopathy. Nature genetics 39: 1134-1139

Penga L, Rasmussena MI, Chailyana A, Houenb G \& Højrupa P (2014) Probing the structure of human protein disulfide isomerase by chemical cross-linking combined with mass spectrometry. J Proteomics 108: 1-16

Pimentel MR, Falcone S, Cadot B \& Gomes ER (2017) In Vitro Differentiation of Mature Myofibers for Live Imaging. Journal of visualized experiments: JoVE: e55141-e55141

Pizon V, Gerbal F, Diaz CC \& Karsenti E (2005) Microtubule-dependent transport and organization of sarcomeric myosin during skeletal muscle differentiation. EMBO J. 24: 3781-3792

Qaisar R (2012) Myonuclear Organization and Regulation of Muscle Contraction in Single Muscle Fibres: Effects of Ageing, Gender, Species, Endocrine Factors and Muscle Size. : 1-64

Qaisar R \& Larsson L (2014) What determines myonuclear domain size? Indian J. Physiol. Pharmacol. 58: 1-12

Quon E, Sere YY, Chauhan N, Johansen J, Sullivan DP, Dittman JS, Rice WJ, Chan RB, Di Paolo G, Beh CT \& Menon AK (2018) Endoplasmic reticulum-plasma membrane contact sites integrate sterol and phospholipid regulation. PLoS Biol. 16: e2003864-41

Rabai A, Reisser L, Reina-San-Martin B, Mamchaoui K, Cowling BS, Nicot A-S \& Laporte J (2019) Allele-Specific CRISPR/Cas9 Correction of a Heterozygous DNM2 Mutation Rescues Centronuclear Myopathy Cell Phenotypes. Mol Ther Nucleic Acids 16: 246-256

Ralston E, Lu Z \& Ploug T (1999) The organization of the Golgi complex and microtubules in skeletal muscle is fiber type-dependent. J. Neurosci. 19: 10694-10705

Robson MI, Las Heras de JI, Czapiewski R, Lê Thành P, Booth DG, Kelly DA, Webb S, Kerr ARW \& Schirmer EC (2016) Tissue-Specific Gene Repositioning by Muscle Nuclear Membrane Proteins Enhances Repression of Critical Developmental Genes during Myogenesis. Mol. Cell 62: 834-847

Roman W, Martins JP, Carvalho FA, Voituriez R, Abella JVG, Santos NC, Cadot B, Way M \& Gomes ER (2017) Myofibril contraction and crosslinking drive nuclear movement to the periphery of skeletal muscle. Nat. Cell Biol. 152: 1376-33 
Romero NB \& Bitoun M (2011) Centronuclear myopathies. Semin Pediatr Neurol 18: 250-256

Romero NB \& Laporte J (2013) Centronuclear Myopathies Oxford, UK: Wiley-Blackwell

Sampath SC, Sampath SC \& Millay DP (2018) Myoblast fusion confusion: the resolution begins. : 1-10

Satoh M, Shimada A, Keino H, Kashiwai A, Nagai N, Saga S \& Hosokawa M (2005) Functional characterization of 3 thioredoxin homology domains of ERp72. Cell Stress Chaperones 10: 278-284

Schroeder B, Weller SG, Chen J, Billadeau D \& McNiven MA (2010) A Dyn2-CIN85 complex mediates degradative traffic of the EGFR by regulation of late endosomal budding. EMBO J. 29: 3039-3053

Schulze RJ, Weller SG, Schroeder B, Krueger EW, Chi S, Casey CA \& McNiven MA (2013) Lipid droplet breakdown requires dynamin 2 for vesiculation of autolysosomal tubules in hepatocytes. J. Cell Biol. 203: 315-326

Straube A \& Merdes A (2007) EB3 regulates microtubule dynamics at the cell cortex and is required for myoblast elongation and fusion. Current biology: CB 17: 1318-1325

Sun Y, Leong NT, Wong T \& Drubin DG (2015) A Pan1/End3/Sla1 complex links Arp2/3-mediated actin assembly to sites of clathrin-mediated endocytosis. Mol. Biol. Cell 26: 3841-3856

Tanabe K \& Takei K (2009) Dynamic instability of microtubules requires dynamin 2 and is impaired in a CharcotMarie-Tooth mutant. J. Cell Biol. 185: 939-948

Tassin AM, Paintrand M, Berger EG \& Bornens M (1985) The Golgi apparatus remains associated with microtubule organizing centers during myogenesis. J. Cell Biol. 101: 630-638

Thompson HM, Cao H, Chen J, Euteneuer U \& McNiven MA (2004) Dynamin 2 binds gamma-tubulin and participates in centrosome cohesion. Nat. Cell Biol. 6: 335-342

Wada I, Rindress D, Cameron PH, Ou WJ, Doherty JJ, Louvard D, Bell AW, Dignard D, Thomas DY \& Bergeron JJ (1991) SSR alpha and associated calnexin are major calcium binding proteins of the endoplasmic reticulum membrane. J. Biol. Chem. 266: 19599-19610

Wang S, Reuveny A \& Volk T (2015) Nesprin provides elastic properties to muscle nuclei by cooperating with spectraplakin and EB1. J. Cell Biol. 209: 529-538

Wang Z, Cui J, Wong WM, Li X, Xue W, Lin R, Wang J, Wang P, Tanner JA, Cheah KSE, Wu W \& Huang J-D (2013) Kif5b controls the localization of myofibril components for their assembly and linkage to the myotendinous junctions. Development 140: 617-626

Wilmshurst JM, Lillis S, Zhou H, Pillay K, Henderson H, Kress W, Müller CR, Ndondo A, Cloke V, Cullup T, Bertini E, Boennemann C, Straub V, Quinlivan R, Dowling JJ, Al-Sarraj S, Treves S, Abbs S, Manzur AY, Sewry CA, et al (2010) RYR1 mutations are a common cause of congenital myopathies with central nuclei. Annals of neurology 68: 717-726

Wilson MH \& Holzbaur ELF (2014a) Nesprins anchor kinesin-1 motors to the nucleus to drive nuclear distribution in muscle cells. Development 142: 218-228

Wilson MH \& Holzbaur ELF (2014b) Nesprins anchor kinesin-1 motors to the nucleus to drive nuclear distribution in muscle cells. Development 142: 218-228

Zhang J, Zheng X, Yang X \& Liao K (2009) CIN85 associates with endosomal membrane and binds phosphatidic acid. Cell Research 19: 733-746 
bioRxiv preprint doi: https://doi.org/10.1101/2020.05.04.076208; this version posted May 5, 2020. The copyright holder for this preprint (which was not certified by peer review) is the author/funder. All rights reserved. No reuse allowed without permission.

Figure 1

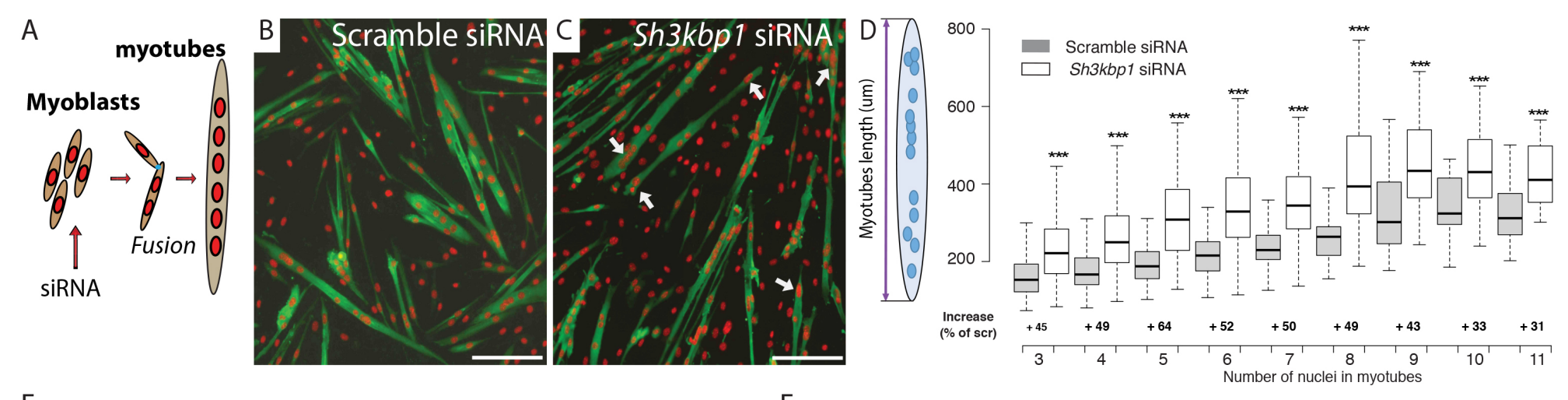

$\mathrm{E}$
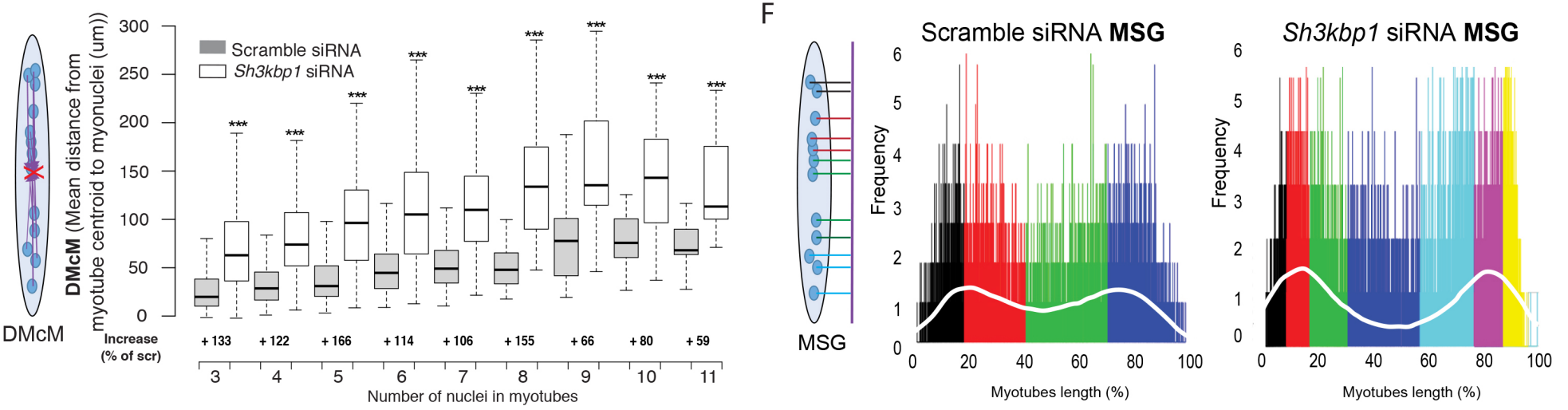
bioRxiv preprint doi: https://doi.org/10.1101/2020.05.04.076208; this version posted May 5, 2020. The copyright holder for this preprint (which was not certified by peer review) is the author/funder. All rights reserved. No reuse allowed without permission.

Figure 2
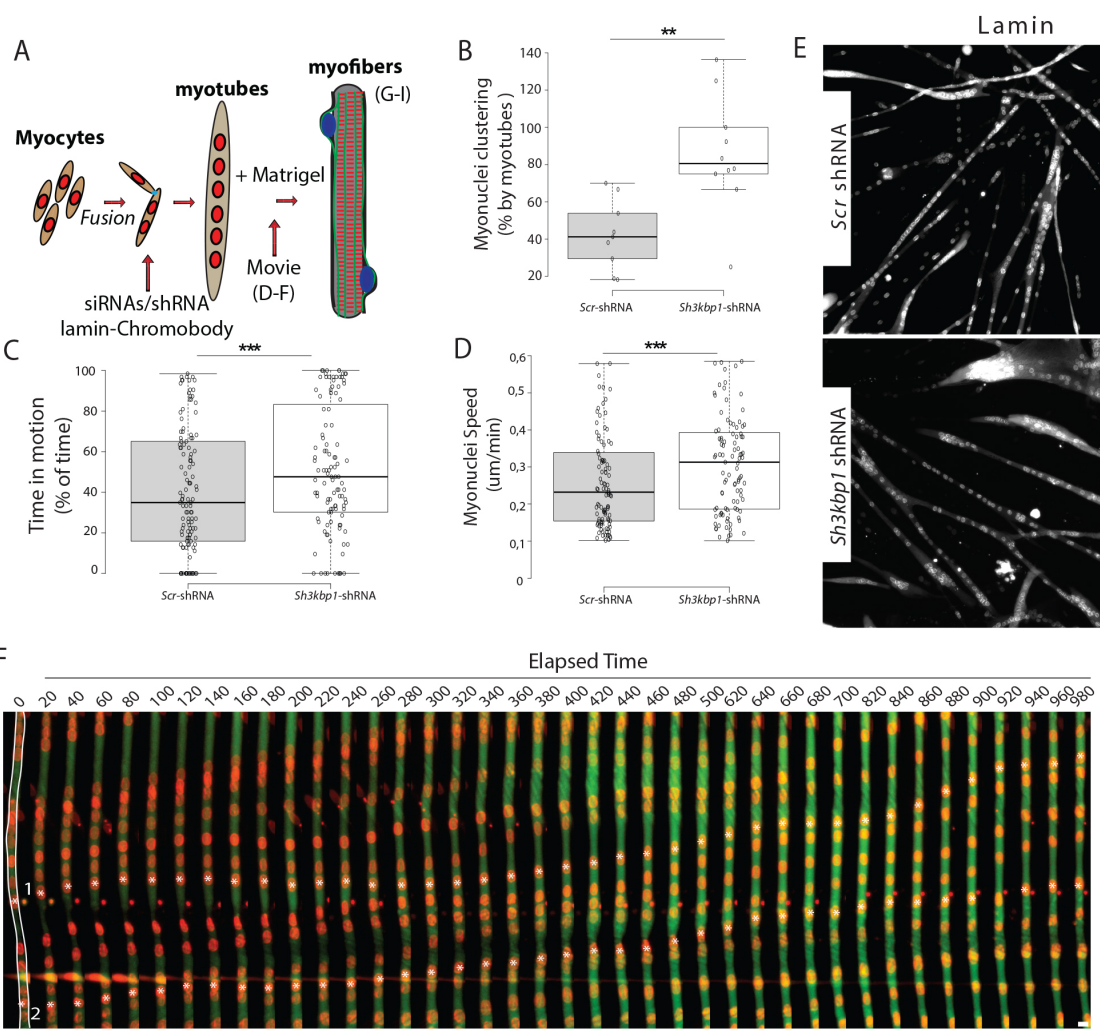

Elapsed Time

G


$\mathrm{H}$

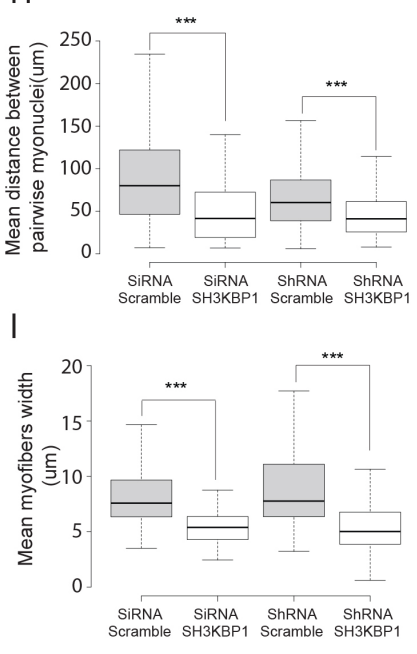


bioRxiv preprint doi: https://doi.org/10.1101/2020.05.04 076208; this version posted May 5, 2020. The copyright holder for this preprint (which was not certified by peer review) is the author/funder. All rights reserved. No reuse allowed without permission.

\section{Figure 3}

A

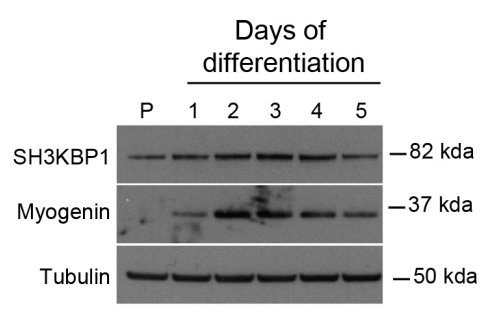

$\mathrm{E}$
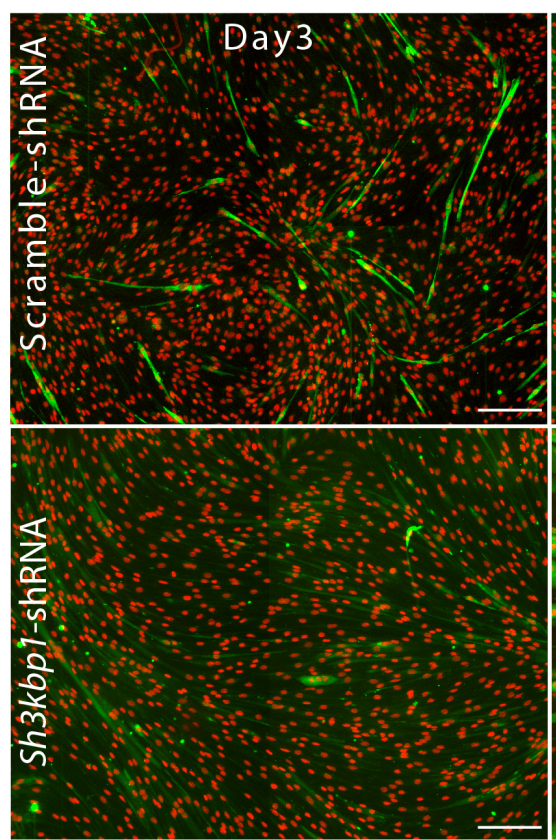

B
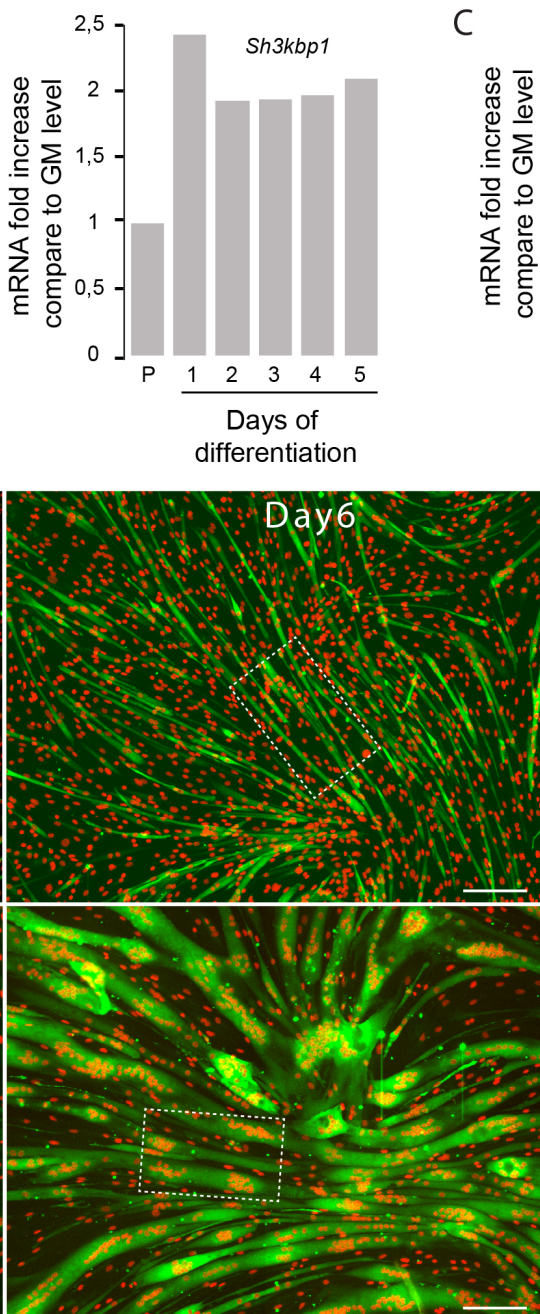

C

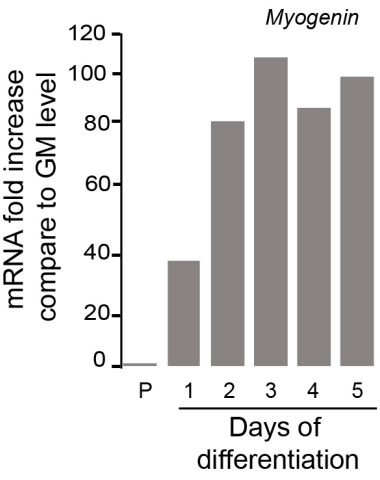

D

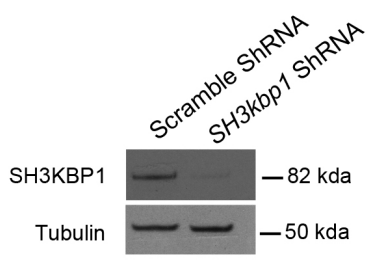

Scramble- $\quad$ Sh3kbp 1ShRNA shRNA



Number of myonuclei by clusters 
Figure 4

C2C12 myoblast culture

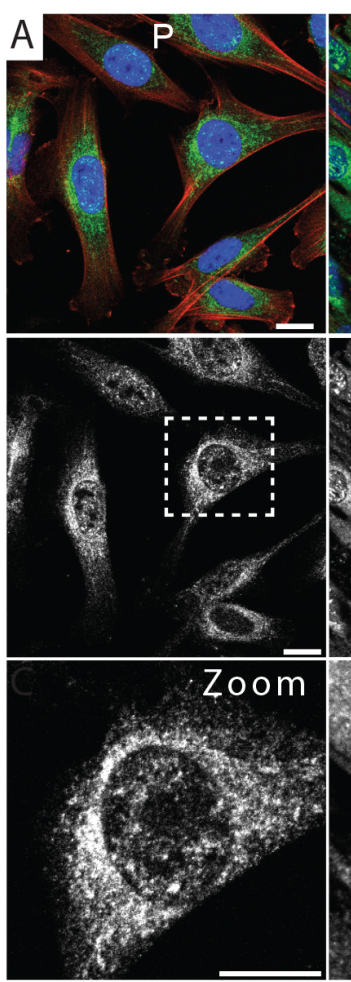

$\frac{r}{n}$
$u$
$u$
$u$
$\frac{1}{c}$
$\frac{1}{n}$
$\frac{0}{0}$
$\frac{1}{m}$
$\frac{1}{n}$

$$
\text { FL }
$$

\begin{tabular}{ll|l|l|l|l|l|l|l|l|} 
& A & B & C & Pro & Ser CC
\end{tabular} N-term A $\quad$ B $\quad$ C

C-term

Pro Ser cc

sC-term
Diff Day 5



Primary myoblast culture
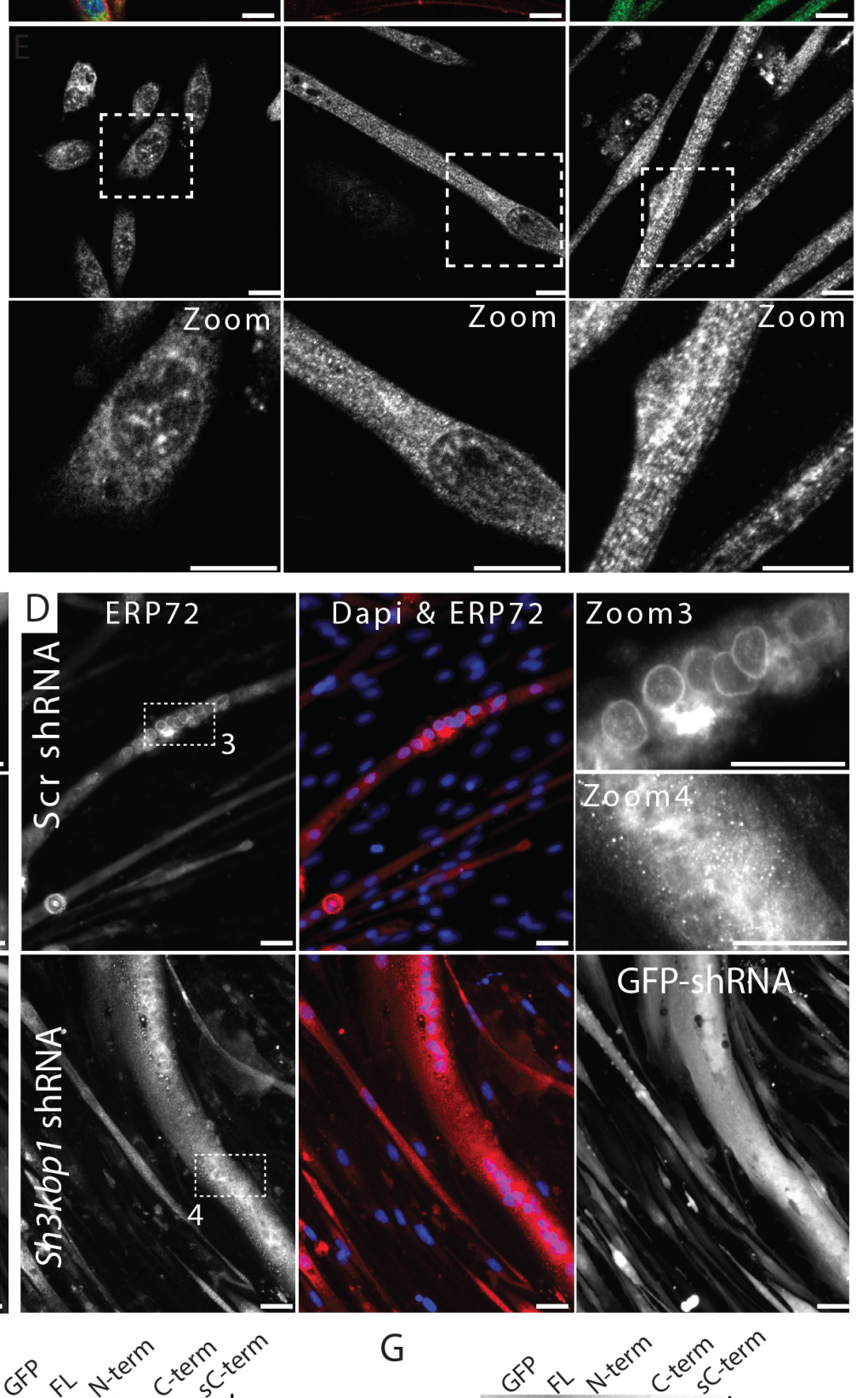

F

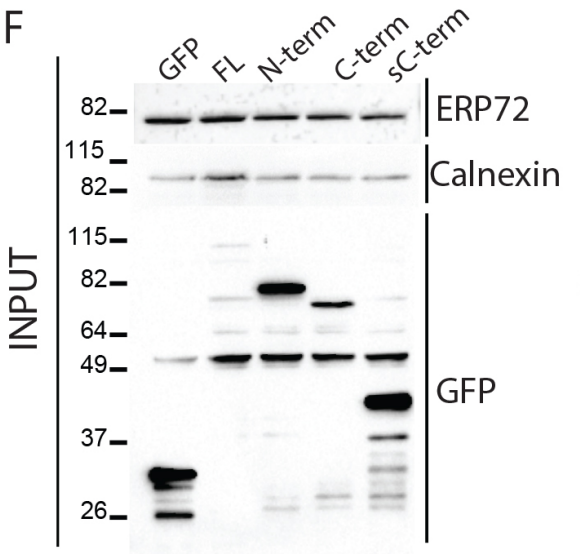

G

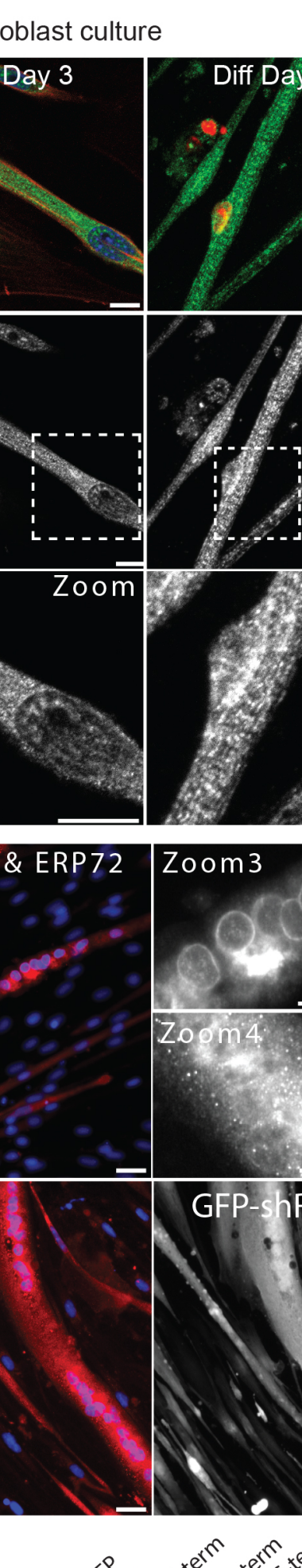

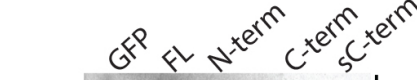

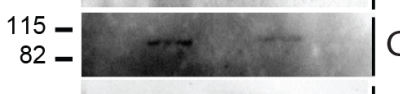

ERP72

으

$115-$

Calnexin

64

49 -

12.

GFP 


\section{Figure 5}
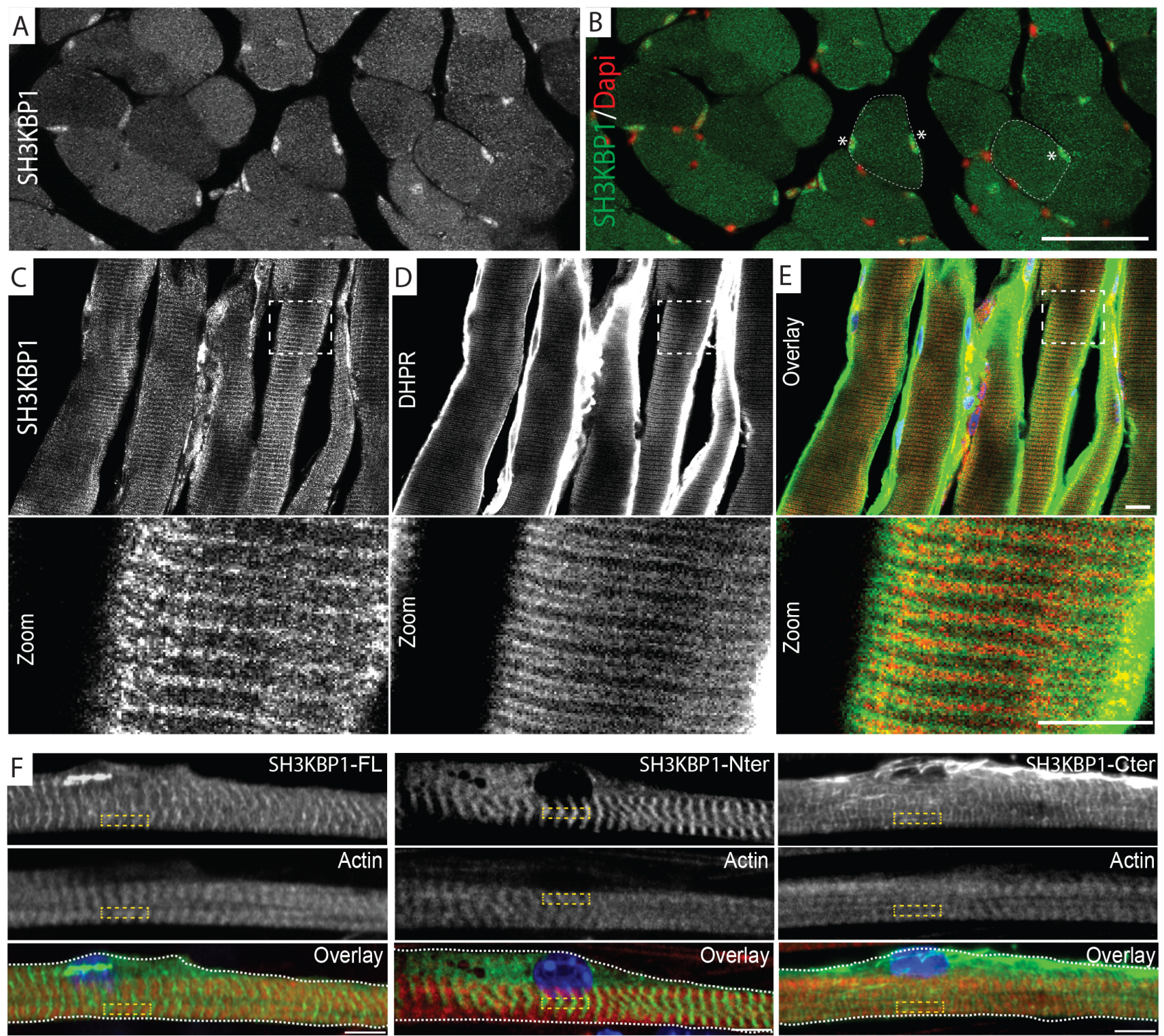

- SH3KBP1-Nter

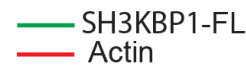

- Actin

- $\mathrm{SH} 3 \mathrm{KBP} 1-\mathrm{Cter}$

G
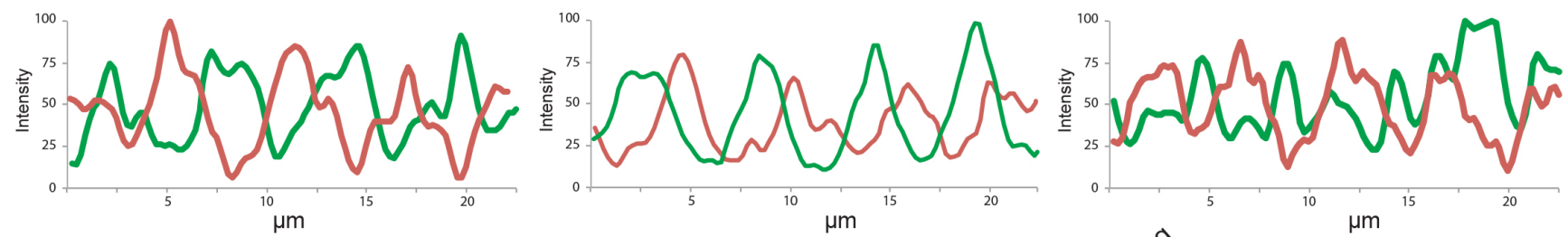

$\mathrm{H}$
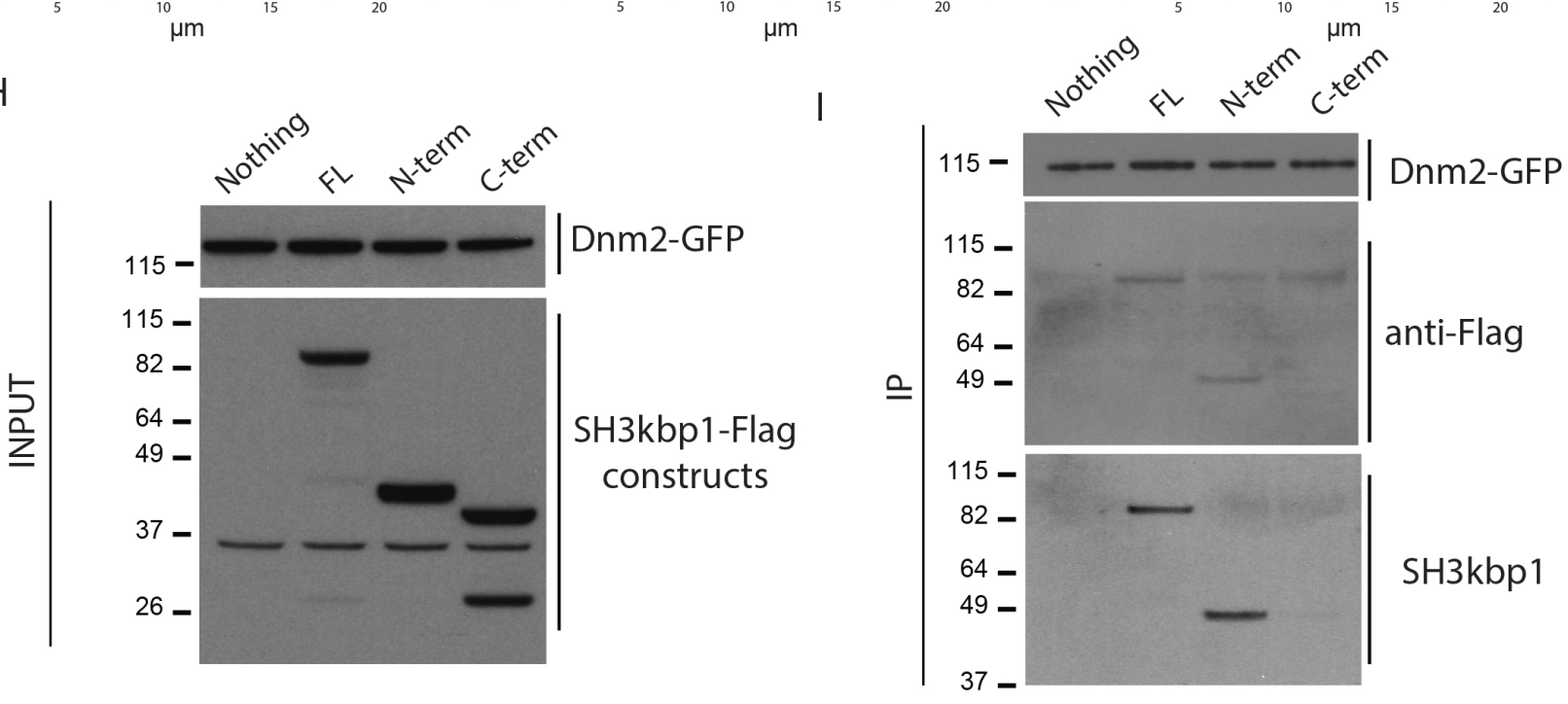
bioRxiv preprint doi: https://doi.org/10.1101/2020.05.04 076208; this version posted May 5, 2020. The copyright holder for this preprint (which was not certified by peer review) is the author/funder. All rights reserved. No reuse allowed without permission.

Figure 6

bioRxiv preprint doi: https://doi.org/10.1101/2020.05.04.076208; this version posted May 5, 2020. The copyright holder for this preprint (which was not certified by peer review) is the author/funder. All rights reserved. No reuse allowed without permission.

Figure 7

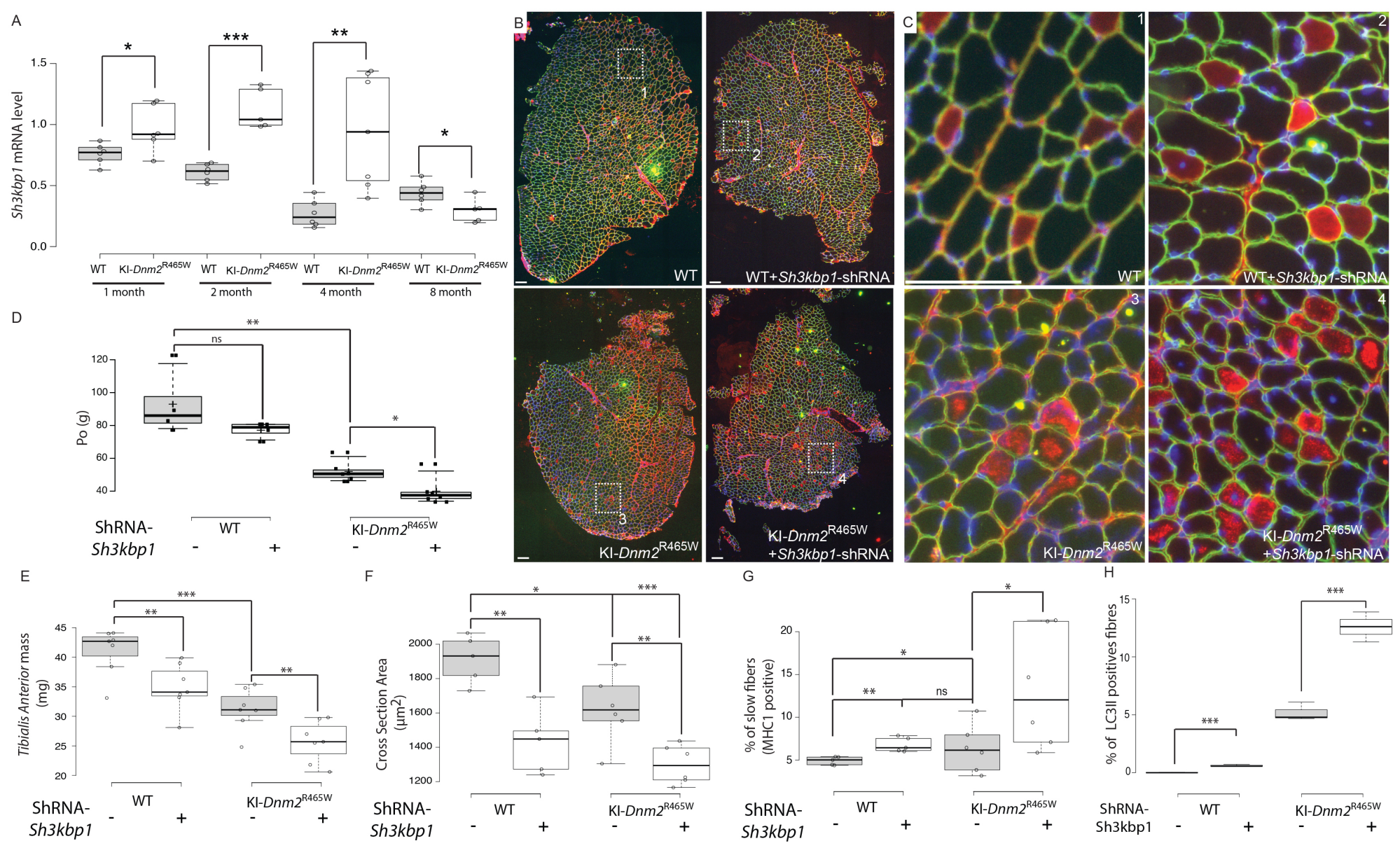




\section{Supplementary Figure 1}
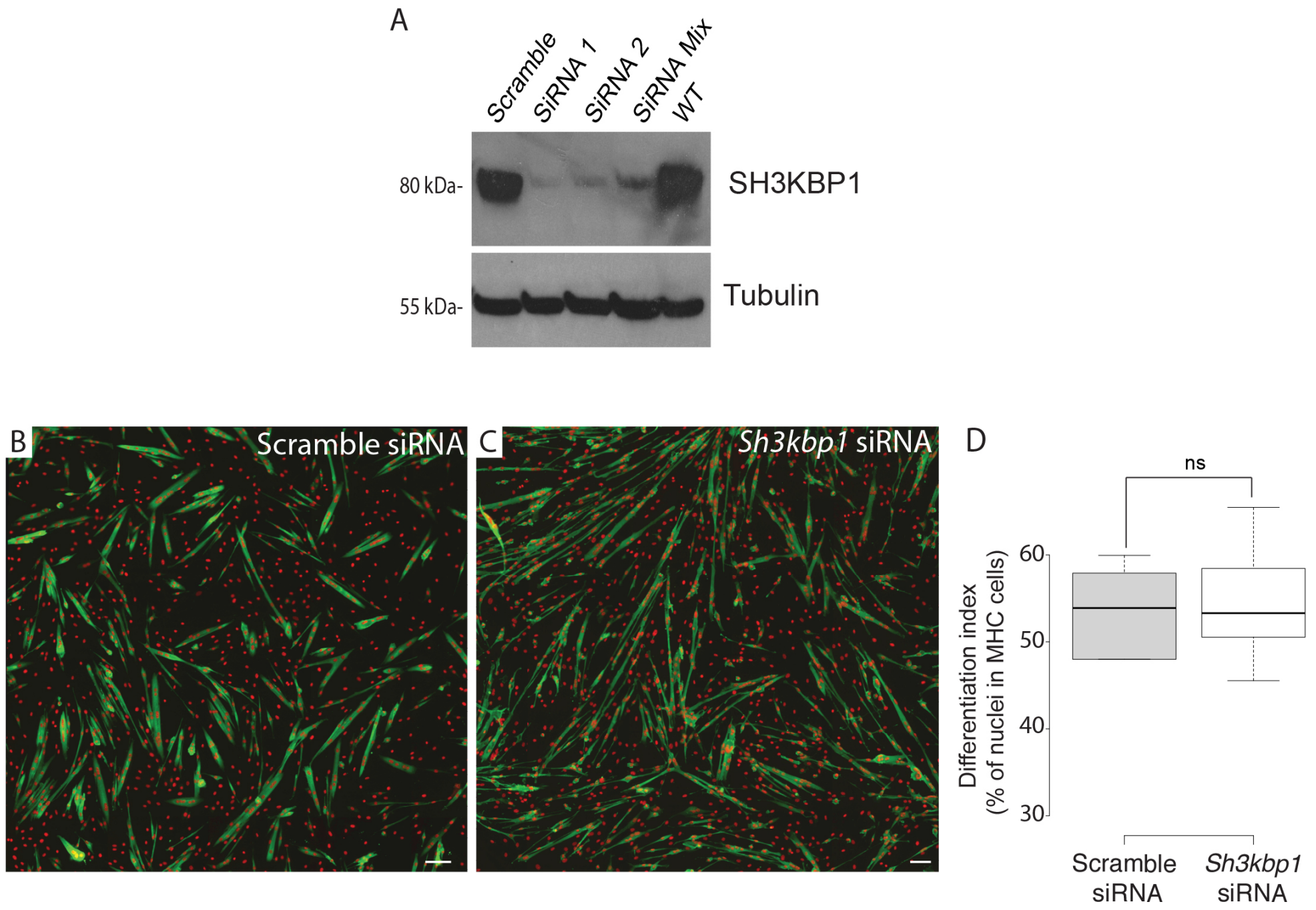

E

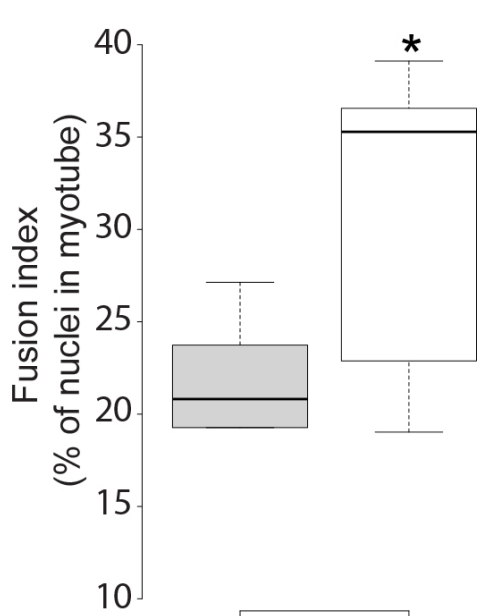

Scramble Sh3kbp 1 SIRNA SiRNA
F

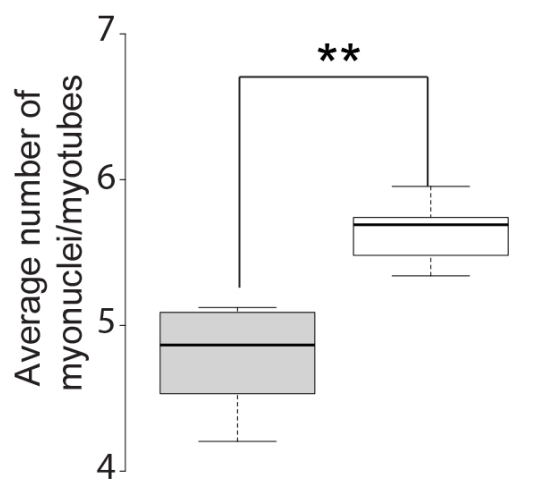

Scramble Sh3kbp 1 siRNA siRNA
G
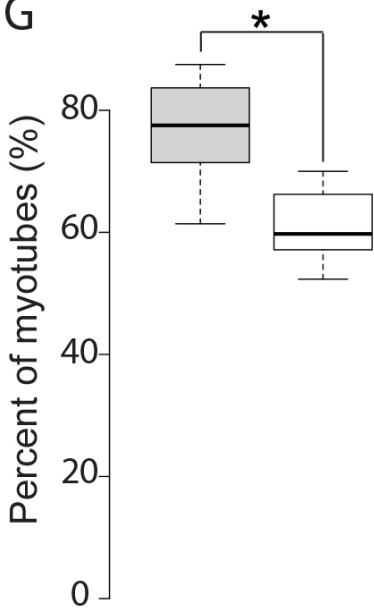

3-5
Scramble siRNA

Sh3kbp1 siRNA
6-10
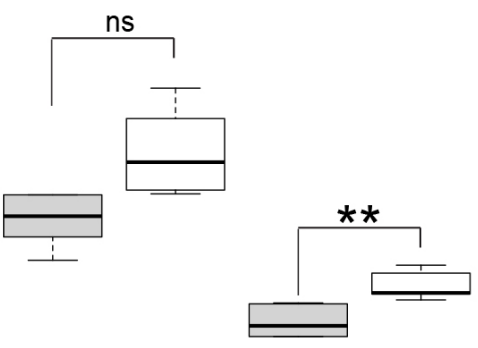

more than 10

Number of nuclei in myotubes 


\section{Supplementary Figure 2}

A

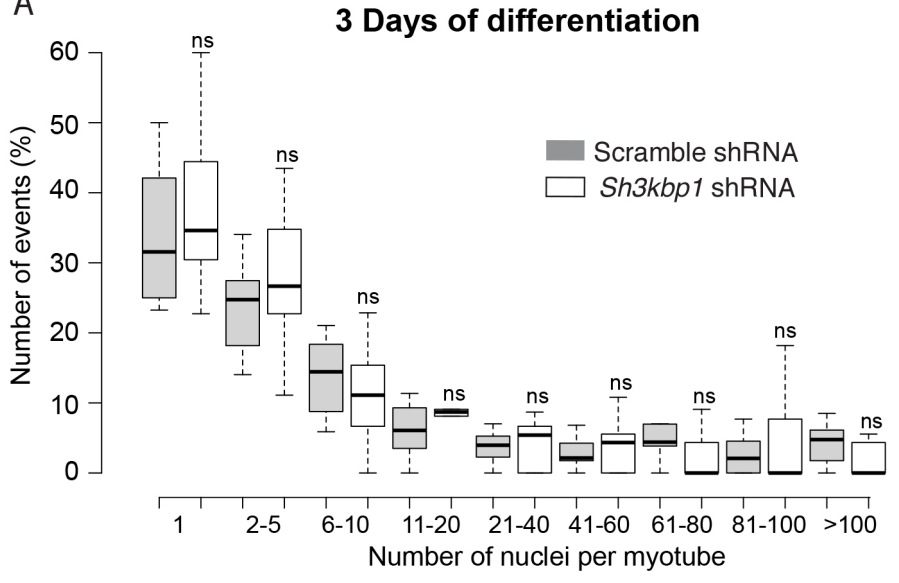

B



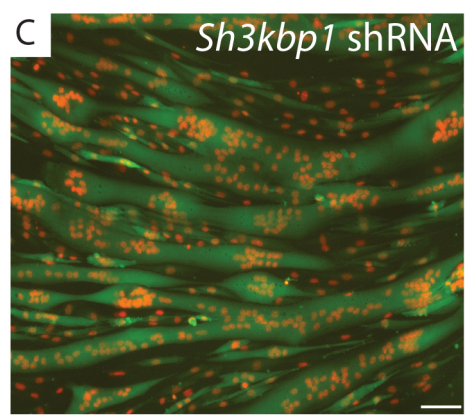


E Ramdon Dots of DHPR



Longitudinally Organized Dots of DHPR



dilly/transversely

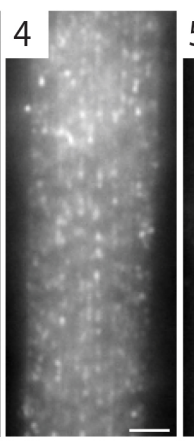
Organized Dots of DHPR

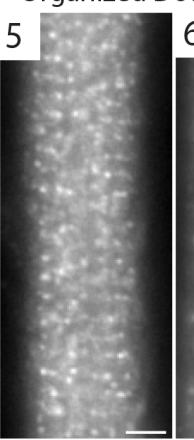






\section{Supplementary Figure 3}

A

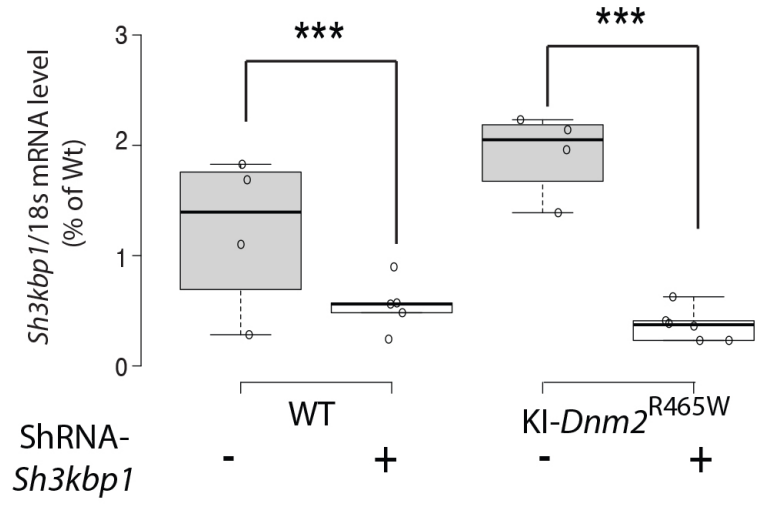

C



Categories of Cross Section Area (um²)

$\mathrm{E}$
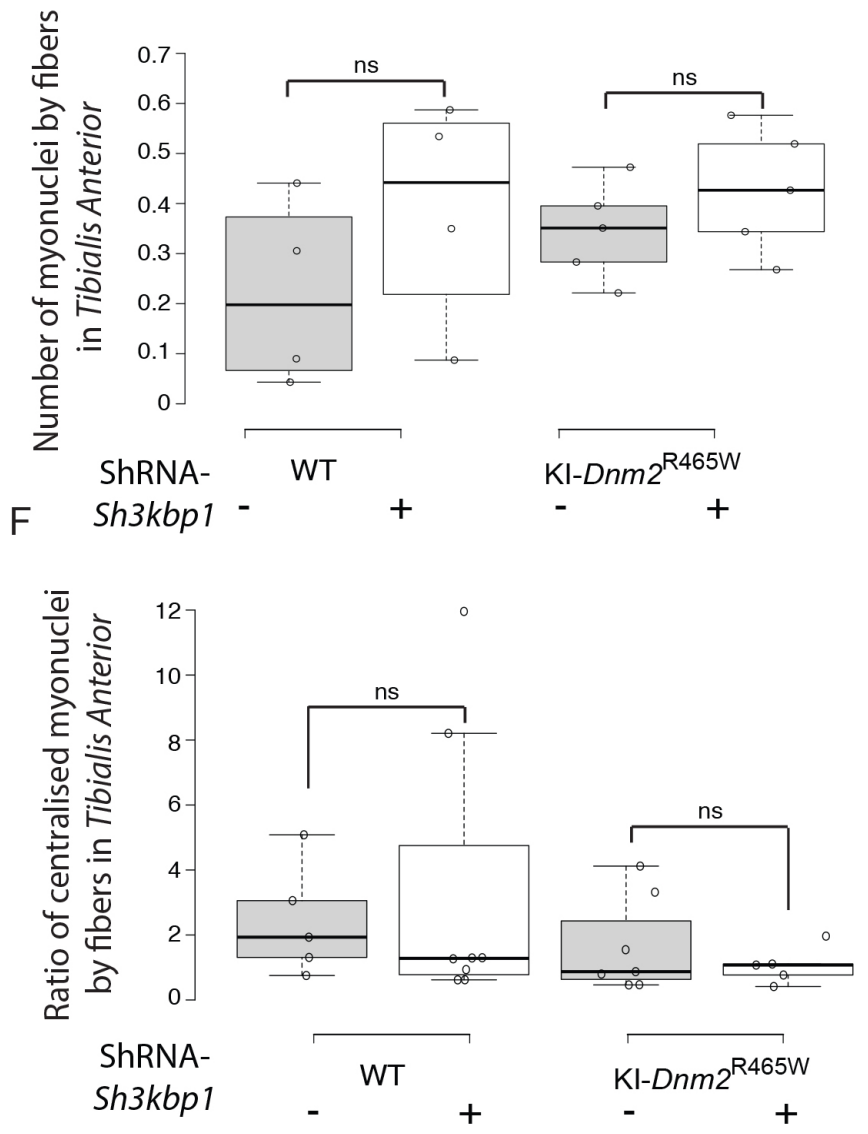

D



B
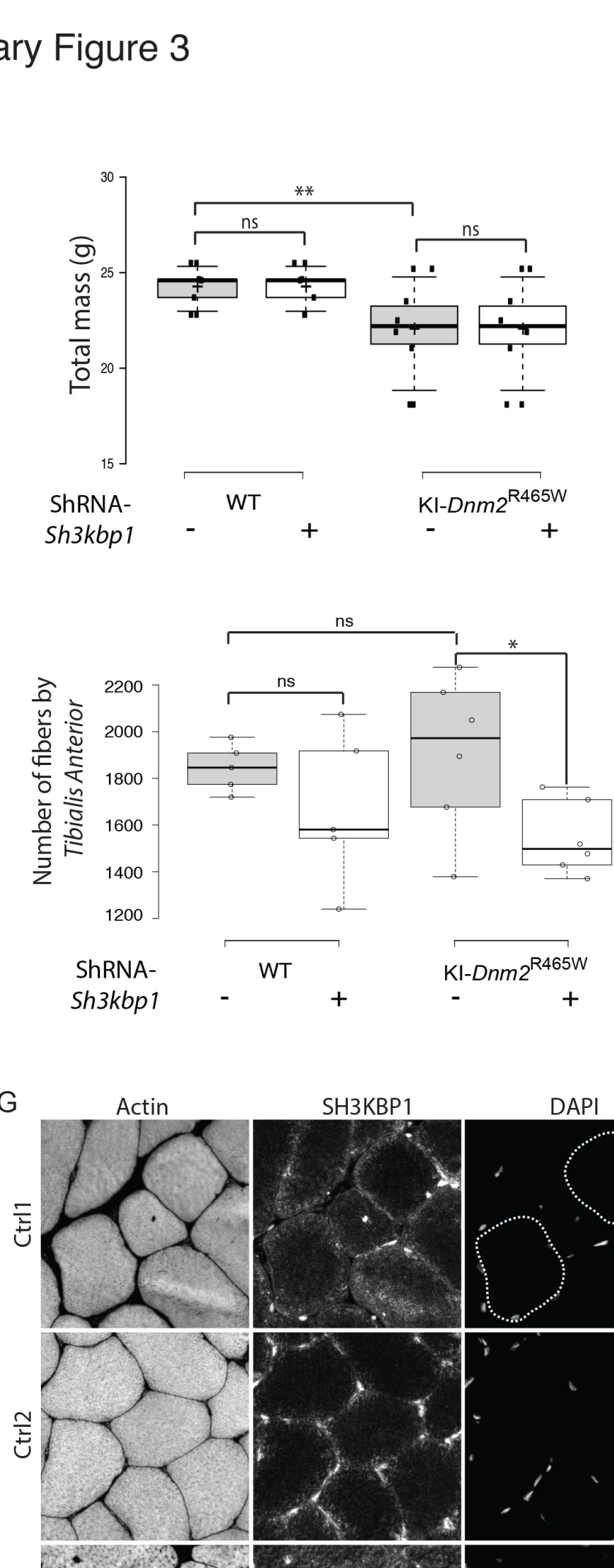

ShRNASh $3 k b p 1$

G

$\bar{\Xi}$ Actin

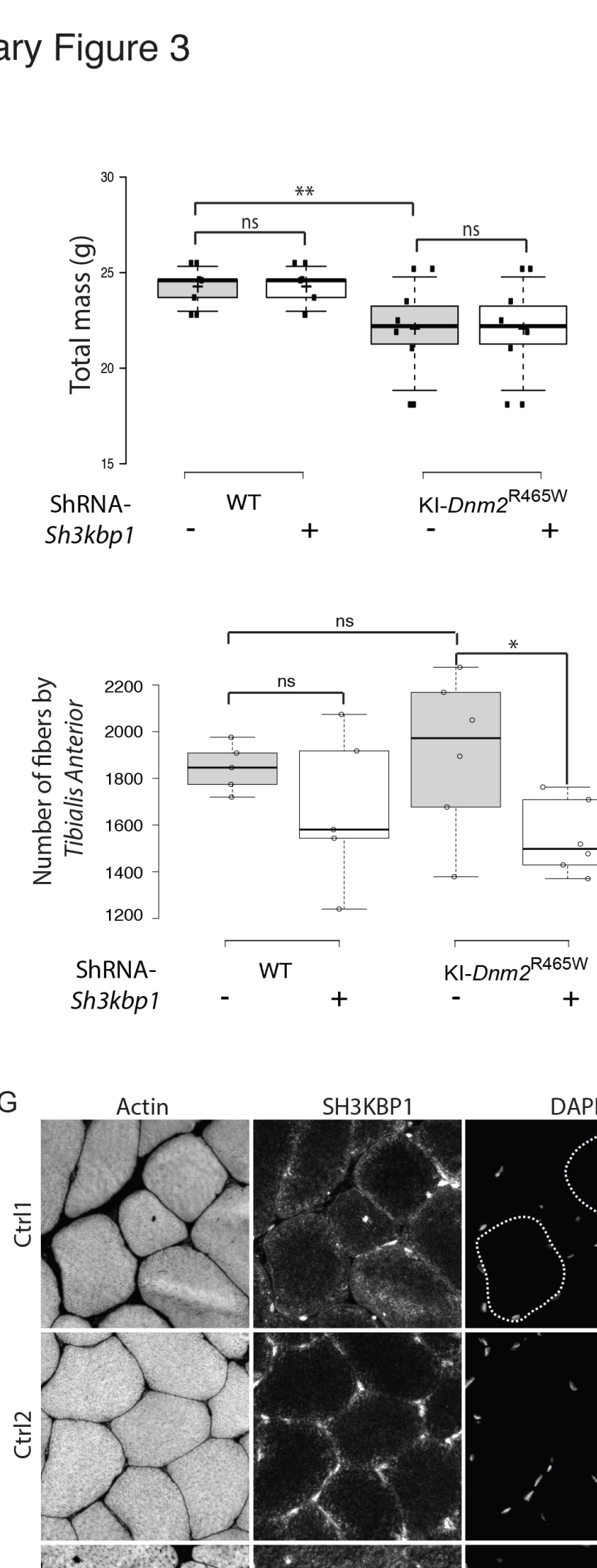

$\mathrm{KI}-\mathrm{Dnm} 2^{\mathrm{R} 465 \mathrm{~W}}$
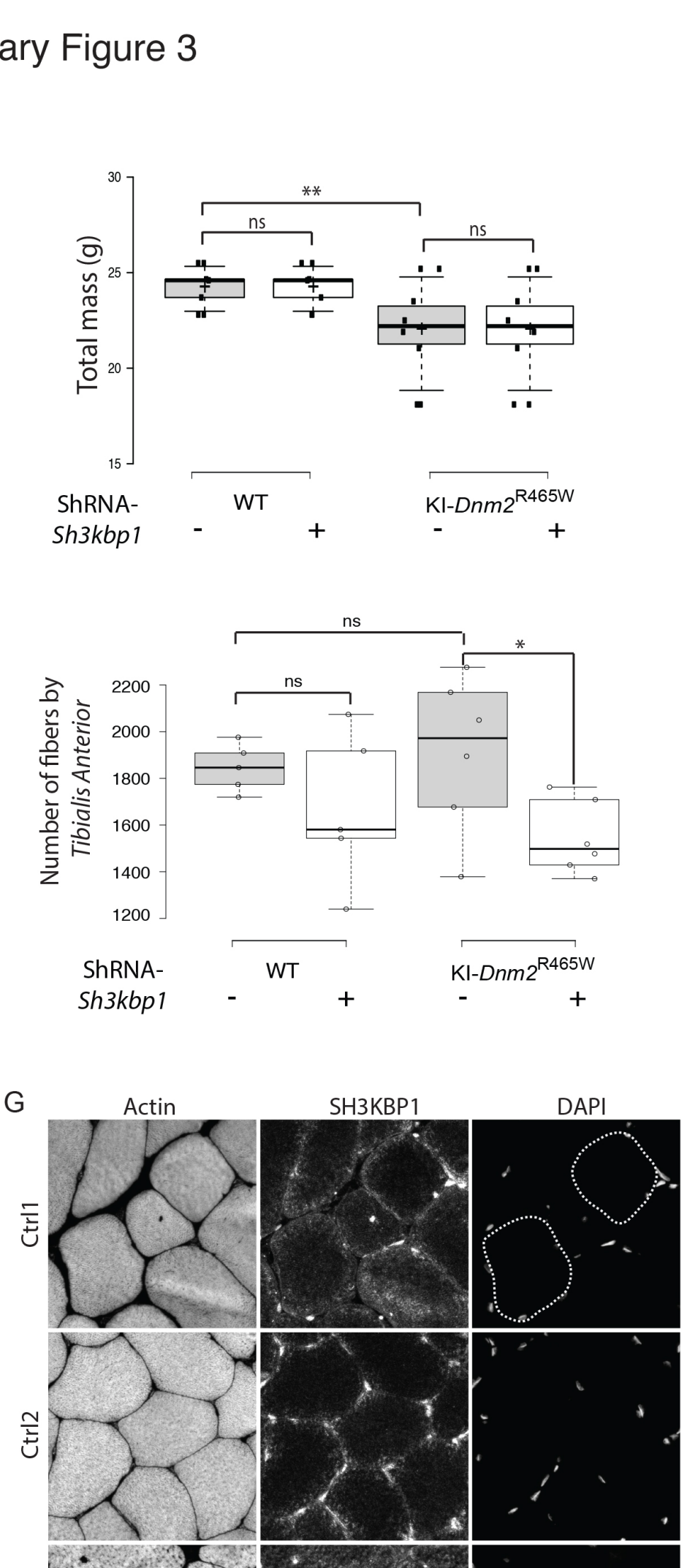

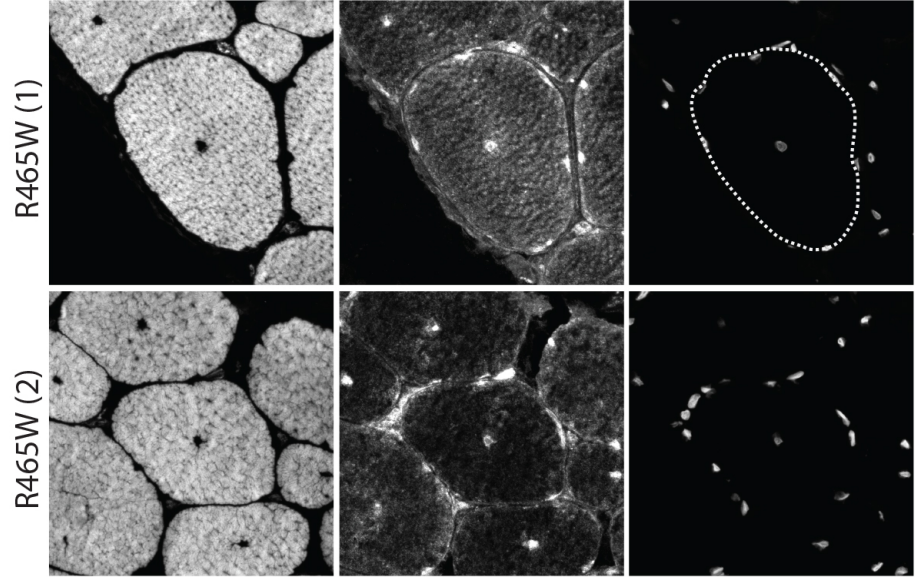




\section{Supplementary Table 1}

siRNA/shRNA sequence

\begin{tabular}{|c|c|c|}
\hline siRNA & Sense oligonucleotide sequence & Anti-Sense oligonucleotide sequence \\
\hline SH3KBP1 \#1 & GGUUGUAGGAGAGGUAGAGtt & CUCUACCUCUCCUACAACCtc \\
\hline SH3KBP1 \#2 & GGUUUUGACUCUGUGAUAUtt & AUAUCACAGAGUCAAAACCtt \\
\hline SH3KBP1 \#3 & GGUCGAUUGAAGUGGAAAAtt & UUUUCCACUUCAAUCGACCtt \\
\hline shRNA & Clone Name & Target Sequence \\
\hline SH3KBP1 & MSH032547-2-CU6(OS308398) & ttacctccagctacatcaa \\
\hline
\end{tabular}

\section{DNA constructs}

\begin{tabular}{|c|c|}
\hline Name & Company/collaborators \\
\hline SH3KBP1-Full length-GFP & Buchman VL Lab Gift \\
\hline SH3KBP1-Full length-M2 & Buchman VL Lab Gift \\
\hline SH3KBP1-C-terminal-GFP & Buchman VL Lab Gift \\
\hline SH3KBP1- C-terminal -M2 & Buchman VL Lab Gift \\
\hline SH3KBP1- N-terminal -GFP & Buchman VL Lab Gift \\
\hline SH3KBP1-N-terminal -M2 & Buchman VL Lab Gift \\
\hline SH3KBP1-CC -GFP & Buchman VL Lab Gift \\
\hline SH3KBP1-CC -M2 & Laporte J Lab Gift \\
\hline DNM2-GFP &
\end{tabular}

Primers sequences

\begin{tabular}{|c|c|}
\hline Primers & Sequence \\
\hline SH3KBP1 F & AATACCGGTTCTTCCTCGGC \\
\hline SH3KBP1 R & CATCCTCCACCAACTCGGAC \\
\hline Gusb F & GAGGATTGCCAACGAAACCG \\
\hline Gusb R & GTGTCTGGGGACCACCTTTGA \\
\hline GAPDH F & AACTTTGGCATTGTGGAAGG \\
\hline GAPDH R & ACACATTGGGGGTAGGAACA \\
\hline RpL4 F & GCCATGAGAGCGAAGTGG \\
\hline RpL4 R & CTCCTGCAGGCGTCGTAG \\
\hline
\end{tabular}

\begin{tabular}{|lll|}
\hline $18 \mathrm{~s}$ & Forward & CGC CGC TAG AGG TGA AAT C \\
& Reverse & CCA GTC GGC ATC GTT TAT GG \\
\hline Sh3Kbp1 & Forward & CCA TGC ACG ATG TAT CCA GTG \\
& Reverse & GTC GTT CTC CTC GTT TAT TGG TT \\
\hline
\end{tabular}




\section{Supplementary Table 2}

\begin{tabular}{|c|c|c|c|}
\hline Antibody or Dye & $\begin{array}{c}\text { Species and } \\
\text { Utility }\end{array}$ & Dilution Factor & Manufacturer and Reference \\
\hline Anti-SH3KBP1 & Rabbit & $1: 100$ & Sigma- HPA003355 \\
\hline Anti-SH3KBP1 (Cin85 H-300) & Rabbit & $1: 1000$ & Santa Cruz, sc-48746 \\
\hline $\begin{array}{l}\text { Anti-SH3KBP1 } \\
\text { (SETA/Cin85/Ruk/SH3KBP1 Antibody } \\
\text { clone 179.1.E1) 05-731 }\end{array}$ & Mouse & 1:1000 & Merck 05-731 \\
\hline Anti-a-Tubulin & Mouse - primary & 1:1000 & $\begin{array}{c}\text { Sigma } \\
\text { T6074-200uL }\end{array}$ \\
\hline Anti-myogenin (F5D) & Mouse & $1: 100$ & Santa Cruz sc-12732 \\
\hline Anti-RCAS1 (D2B6N) & Rabbit & $1: 200$ & Cell Signaling \#12290 \\
\hline Anti-ERP72 (D70D12) & Rabbit & $1: 100$ & Cell Signaling \#5033 \\
\hline Anti-laminin & Rat & $1: 200$ & Sigma \\
\hline Anti-GFP (3H9) & Rat & $1: 1000$ & Chromotek \\
\hline Anti-Flag M2 & Mouse & $1: 1000$ & Sigma F1804 \\
\hline Anti-Calnexin (C5C9) & Rabbit & $1: 100$ & Cell Signaling \#2679 \\
\hline Anti-LC3II/LC3I & Rabbit - primary & $1: 250$ & Sigma L7543 \\
\hline Anti-Myosin skeletal slow & Mouse & $1: 100$ & Sigma M8421 \\
\hline MF20 & Mouse-primary & $1: 10$ & DSHB \\
\hline Anti-DHPR & Rabbit & $1: 10$ & DSHB (IIID5E1) \\
\hline Dapi-brilliant blue & Dye & $1: 50000$ & $\begin{array}{l}\text { Thermo Fisher Scientifics } \\
\text { D1306 }\end{array}$ \\
\hline Phalloidin-Alexa Fluorr 647 & Dye & $1: 100$ & $\begin{array}{l}\text { Thermo Fisher Scientifics } \\
\text { A22287 }\end{array}$ \\
\hline Anti-mouse-HRP & Goat - secondary & $1: 3000$ & $\begin{array}{c}\text { Invitrogen } \\
62-6520\end{array}$ \\
\hline Anti-rabbit-HRP & $\begin{array}{c}\text { Goat - } \\
\text { secondary }\end{array}$ & $1: 3000$ & $\begin{array}{l}\text { Invitrogen } \\
65-6120\end{array}$ \\
\hline $\begin{array}{l}\text { Anti-rat- } \\
\text { Alexa Fluor } 488\end{array}$ & $\begin{array}{l}\text { Donkey - } \\
\text { secondary }\end{array}$ & $1: 500$ & $\begin{array}{l}\text { Thermo Fisher Scientifics } \\
\text { A-21208 }\end{array}$ \\
\hline $\begin{array}{l}\text { Anti-rabbit- } \\
\text { Alexa Fluor } 647\end{array}$ & $\begin{array}{c}\text { Goat - } \\
\text { secondary }\end{array}$ & $1: 500$ & $\begin{array}{l}\text { Thermo Fisher Scientifics } \\
\text { A-21245 }\end{array}$ \\
\hline $\begin{array}{l}\text { Anti-mouse- } \\
\text { Alexa Fluor } 647\end{array}$ & $\begin{array}{c}\text { Goat - } \\
\text { secondary }\end{array}$ & $1: 500$ & $\begin{array}{l}\text { Thermo Fisher Scientifics } \\
\text { A-21240 }\end{array}$ \\
\hline
\end{tabular}

